\title{
Distribución espacial y temporal de macroinvertebrados acuáticos en la quebrada La Cascajosa - Garzón (Huila)
}

\section{Spatial and Temporary Distribution of Aquatic Macro-Invertebrates in La Cascajosa Brook}

\author{
Natalia Karolina Portilla Arcos \\ Magister en Ecología y Gestión de Ecosistemas estratégicos \\ nakabio@hotmail.com
}

\section{Resumen}

Se realizó un estudio de la distribución espacial y temporal de macroinvertebrados acuáticos en la quebrada La Cascajosa ubicada en el municipio de Garzón, Huila, entre noviembre y diciembre de 2011 y enero y julio de 2012.

Se colectaron 891 individuos pertenecientes a 5 clases, 13 órdenes, 29 familias, 33 géneros. En cuanto a número de individuos, el Orden Diptera fue el más representativo con 212 seguido de Ephemeroptera con 178, Trichoptera y Coleoptera con 158 y 115 individuos respectivamente. De las clases, Annelida, Mollusca, Crustacea y Platelminta, fueron hallados en menor proporción (15\%). Al comparar la abundancia de organismos en las estaciones de La quebrada La Cascajosa, se encontró similitud entre las estaciones 1 y 2 , en donde se concentró el 28 y $27 \%$ del total de los individuos registrados se presentó un aumento en el número de individuos en la estación 3 alcanzando un 39,3\% del total de la población, y en la estación 4 este número disminuye. El Índice de Shannon-Wiener indicó que en la quebrada hay una mediana diversidad para la fauna de macroinvertebrados en las estaciones $1,2,3$ y baja diversidad en la estación 4 .

La dominancia en las comunidades de macroinvertebrados acuáticos estuvo representada por los órdenes Megaloptera y Coleoptera debido al tamaño de los organismos respecto al espacio abarcado. Mayor dominancia en estaciones 3 y 4 , estaciones que fueron las de más baja diversidad. De acuerdo con el BMWP, La estación I tiene aguas clase II buena calidad, las demás estaciones presentan agua Clase III, aceptable (ligeramente contaminada) el valor más bajo para éste índice lo obtuvo la estación 4.

Mediante el análisis de las variables físicas y químicas se determinó que la quebrada La Cascajosa presenta un buen nivel de conservación y que cumple con las condiciones mínimas requeridas para el desarrollo de la vida.

\section{Abstract}

A study of spatial and temporary distribution of macro-invertebrates at the Cascajosa Brook, located in the town of Garzón, Huila, was carried out between November and December of 2011 and January and July of 2012.

891 individuals were collected belonging to 5 kinds, 13 orders, 29 families, 33 genres. In terms of the number of individuals, the Diptera Order was the most representative with 212 followed by Ephemeropterous with 178, Trichpterous and Choleoptera with 158 and 115 individuals respectively. From the kinds, Annelida, Mollusca, Crustaceous and Platelminta were found in less proportion (15\%). When comparing the abundance of organisms in the stations of La Cascajosa Brook, a similarity between stations 1 and 2 was found, in which 28 and $27 \%$ of the total registered individuals was concentrated an increase in the number of individuals on station 3 was found, reaching 39\% of the total of the population, and on station 4 this number is reduced. The Shannon-Wiener index indicated that there is a medium diversity on the brook for macro-invertebrate fauna in stations 1,2 , and 3 and low diversity in station 4 .

The dominance in the aquatic macro-invertebrates' communities was represented by the Megalopterous and Choleopterous orders due to the size of the organisms regarding the space reached. Most dominance in stations 3 and 4 , stations which were of the lowest diversity. According to BMWP, station I has kind II waters of good quality, the other stations present Kind III water, acceptable (slightly contaminated) the lowest value for this index was obtained by station 4 .

Through the analysis of physical and chemical variables it was determined that La Cascajosa Brook presents a good level of conservation and that it complies with the minimal conditions required for the development of life.

The Cluster analysis indicated that the places which presented the greatest similarity were stations 1 and 2 with a similarity range above $96 \%$, stations 1,2 and 3 
El análisis de Clúster indicó que los sitios que presentaron mayor similaridad fueron las estaciones 1 y 2 con un rango de similaridad por encima del $96 \%$, Las estaciones 1, 2 y 3 se encuentran más correlacionadas en cuanto a la abundancia, diversidad y riqueza, mostrando mayor porcentaje de similitud las estaciones 1 y 2 para la diversidad y riqueza de especies.

El Análisis Multivariable de Co-inercia, muestra que la información está principalmente resumida por tres ejes. El primero, explica un $61,3 \%$ de la Variabilidad, el segundo un $23,8 \%$ y el tercero un $14,7 \%$ con una correlación de $\mathrm{r}=0.99$.

Palabras clave: Macroinvertebrados, bioindicación, índices bióticos, calidad de agua

\section{Introducción}

La biogeografía, la diversidad y ecología de macroinvertebrados en ríos tropicales son poco conocidas (Jacobsen, 2008). Los macroinvertebrados son los invertebrados superiores a $0,5 \mathrm{~mm}$ de tamaño del cuerpo, comprenden principalmente insectos así como decápodos crustáceos, moluscos, sanguijuelas, oligoquetos y planarias. Son suficientemente grandes como para ser vistos por el ojo humano, la mayoría de ellos viven entre los sedimentos del cauce, y por lo tanto a menudo se refiere a ellos como macrobentos, aunque la mayoría de los insectos tienen un flujo de ciclo de vida anfibiótico y pasan su etapa adulta en la tierra.

El ecosistema de la quebrada La Cascajosa, toma importancia para este estudio ya que comprende por ubicación, zonas rurales y urbanas. Autores como Grimm et al (2008), señalan que las áreas urbanas cubren menos del 3\% de la superficie terrestre del planeta y que las ciudades desempeñan un papel importante en los cambios de la biodiversidad pues la urbanización conduce a la destrucción de los ecosistemas, la fragmentación del hábitat y la extinción de especies. Este ecosistema ha sido muy poco estudiado $y$ no se conoce un registro de la fauna de macroinvertebrados y su distribución en el cuerpo de agua, además es ampliamente intervenido por actividades agrícolas en la zona rural y a lo largo de la quebrada que comprende la zona urbana, su impacto en ella aumenta por los procesos de urbanización y las acciones contaminantes al recurso acuícola que ello signifique.

Grimm et al (2008), también indican que los ecosistemas acuáticos en las zonas urbanas difieren en muchos aspectos de la los naturales, por ejemplo en la hidrología, la morfología, la química del agua y la composición de la flora y la fauna. Esta investigación, tiene el objetivo de evaluar la estructura espaciotemporal de las comunidades de macroinvertebrados acuáticos, respecto a su densidad y número de taxa, relacionadas a variables ambientales en la quebrada La Cascajosa. are more correlated in terms of abundance, diversity and richness, showing the greatest percentage of similarity stations 1 and 2 for the diversity and richness of species.

The Co-Inertia Multivariable Analysis shows that the information is mainly summarized by three axes. The first explains $61,3 \%$ of variability, the second $23,8 \%$ and the third $14,7 \%$ with a correlation of $r=0.99$.

Key Words: macro-invertebrates, bio-indication, biotic indexes, quality of water.

\section{Metodología}

\section{Descripción del área de estudio}

El estudio se efectuó en la quebrada La Cascajosa, ubicada en el departamento del Huila (Colombia), municipio de Garzón. Esta corriente, recorre una distancia aproximada de $2 \mathrm{Km}$ desde su nacimiento (vereda Monserrate, predios del basurero municipal), a 1124 m.s.n.m. y cruzando la zona urbana del municipio a una altitud promedio de 828 m.s.n.m. desde sur oriente (nacimiento) a sur occidente (desembocadura en la quebrada Garzón) (Figura 1). Con base en la clasificación ecológica de Holdridge (1996), el área se caracteriza por ser una zona de vida de Bosque seco tropical (bs-T.); la temperatura media es de $24{ }^{\circ} \mathrm{C}$. Altitud de la cabecera municipal (800 metros sobre el nivel del mar) está ubicado a $2^{\circ} 11^{\prime} 57^{\prime \prime}$ de latitud Norte y $75^{\circ} 38^{\prime} 59^{\prime \prime}$ de longitud Oeste. El municipio goza de toda la variedad de climas (POT 2007).

\section{Selección de las estaciones de muestreo}

Teniendo en cuenta que según Rueda (2002) los hábitats acuáticos y sus asociaciones específicas de invertebrados son muy diversos, por consiguiente la variedad de métodos aplicables para su estudio es muy amplia, y se debe tener en cuenta según este autor, puntos de muestreo representativos tales como nacimiento de cauces, desembocaduras, y zonas de alimentación por tributarios a lo largo del cauce, para abarcar un muestreo representativo que permita describir una aproximación de la composición de la biota presente y sus condiciones ambientales. Esta teoría es también sustentada por autores como Domínguez y Fernández (2009) quienes presentan al detalle las técnicas de muestreo de macroinvertebrados bentónicos y enfatizan en la importancia del ecosistema y tipo de hábitat al ser muestreado.

Estación 1. Está localizada a una altura de $1124 \mathrm{msnm}$. Fue escogida por ser el punto de nacimiento de la quebrada. La vegetación marginal corresponde a bosque ripario en proceso de sucesión, se aprecia un alto grado de intervención agrícola con predominio de 
cultivos de cacao en los dos márgenes. El lecho está constituido por lodos y materia orgánica en descomposición de origen vegetal. El ancho promedio de La quebrada en este punto es de $80 \mathrm{~cm}$ y la profundidad varió entre 4 y $10 \mathrm{~cm}$. (Figura 1).

Estación 2. Se ubica $300 \mathrm{~m}$ después del nacimiento de la quebrada a una altitud de $878 \mathrm{msnm}$. En este sector, la quebrada La Cascajosa es tributada por la quebrada La Cartagena. Debido a que se produce una ligera depresión en el lecho se incrementa la profundidad y con represamiento de las aguas, especialmente en el margen derecho. La profundidad medida fue de 12 a $14 \mathrm{~cm}$ y el ancho de 1,20 $\mathrm{m}$ (Figura 1).

Estación 3. Se localiza en la zona urbana a $1 \mathrm{Km}$ de distancia de la estación anterior. Es la zona con mayor influencia antrópica y mayor intervención. Se encuentra a una altitud de $847 \mathrm{msnm}$, posee una profundidad que varió entre 14 y $36 \mathrm{~cm}$ y ancho de 2,05 m (Figura1)

Estación 4. Esta estación se encuentra una altitud de $800 \mathrm{msnm}$; alcanzó una profundidad entre 20 y $42 \mathrm{~cm}$ y 4,20 m de ancho. Es el punto de desembocadura de la quebrada La Cascajosa en la quebrada Garzón (Figura1).

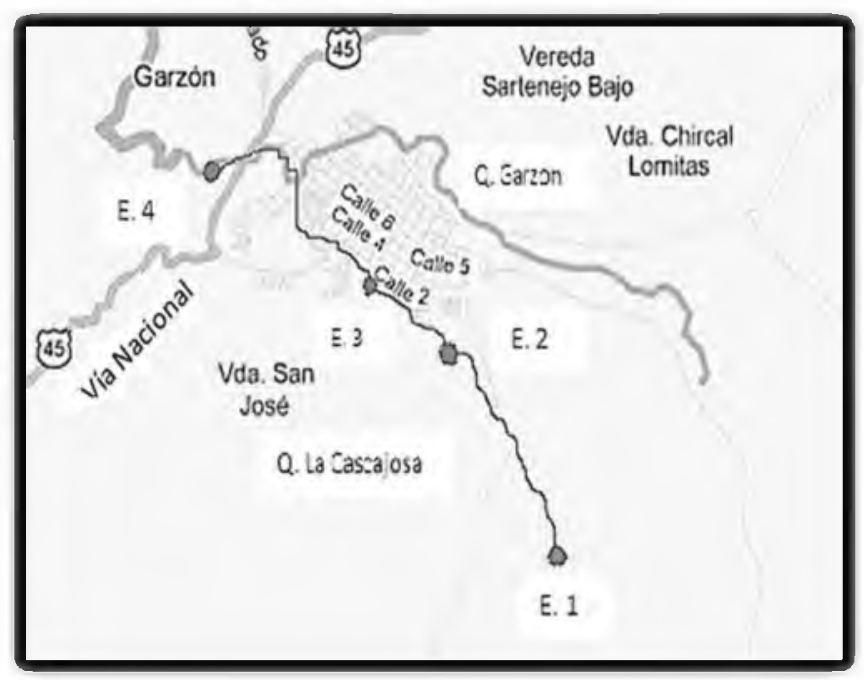

Figura 1. Estaciones de muestreo Quebrada La Cascajosa.

\section{Fase de campo}

Muestreo biológico: Macroinvertebrados bentónicos Para el diseño de muestreo biológico: Macroinvertebrados bentónicos, se tuvo en cuenta:

El tipo de ecosistema o hábitat a ser muestreado, ya que de acuerdo con Baumgaerter et al., (2008), para su diseño se requiere conocer la velocidad de la corriente, la vegetación riparia terrestre y acuática las cuales definen la composición del sustrato en el que se encuentra los invertebrados del bentos.
Representatividad del estudio y esfuerzo de muestreo: Según Rueda (2002) las diferentes subunidades de muestreo y réplicas de una misma subunidad son importantes aunque la dificultad en acceder al sitio de muestreo y tiempo invertido en obtener las muestras hacen difícil esta optimización, por tal razón, el autor sustenta que se debe escoger en la mayoría de los casos entre el tamaño de la muestra (área que se cubrió) y la representatividad de la muestra individual. Otro aspecto que señala a tener en cuenta es el tiempo de muestreo y sugiere que en zonas donde se pueda establecer temporadas de periodo lluvioso y temporadas de periodo seco se recomienda muestrear duran-te los dos periodos para efectos de comparación y deriva. Este aspecto es también tratado por Domínguez y Fernández (2009) y recomiendan muestreos quincenales, mensuales o bimensuales dependiendo del interés del estudio.

Frecuencia del muestreo: Según Junk et al. 1989; Junk y Wantsen, 2004 (citados por Domínguez y Fernández, 2009), en los cuerpos de agua los hábitats funcionales son formados por la hidrología y el pulso de inundación el cual controla los procesos ecológicos, incluyendo las características del ensamble de invertebrados bentónicos. Domínguez y Fernández (2009) proponen que los diseños de muestreo deben considerar las fases de llenado, vaciado y transiciones del ciclo hidrológico. Especifican cuatro momentos característicos del ciclo hidrológico de un río: - Aguas bajas: Período de sequía en el que solo los cauces más profundos mantienen el flujo de agua. - Aguas en ascenso: Inicio del período de lluvias en el que el caudal se incrementa. - Aguas altas: Período de altas lluvias durante el cual se obtienen los máximos caudales. - Aguas en descenso: Finales del período de lluvias.

El autor señala que la intensidad del muestreo depende también de los objetivos del estudio, la capacidad de trabajo, posibilidades logísticas y presupuesto. En cuanto a las redes utilizadas para la captura de especímenes, Buss y Borges (2008) sugieren que redes con tamaño de poro de $500 \mu \mathrm{m}$ puede ser suficiente para capturar las familias más comunes de invertebrados, aunque no sea el tamaño adecuado si el objetivo es analizar larvas de Chironomidae por ejemplo.

De acuerdo con lo anterior, para la presente investigación, durante ocho (8) meses se realizaron cinco (5) muestreos, en cada una de las estaciones; la colecta de macroinvertebrados se efectuó entre las 7:00 a.m. y 12:00 m., en los meses de noviembre y diciembre (registro de lluvias), febrero y marzo (registro de pocas lluvias y transición a temporada seca), y finales de mayo (se registra más tiempo seco y lluvias escasas).

En cada estación, se efectuaron muestreos aproximadamente durante una (1) hora, con tres repeticiones, en un área aproximada de un metro cuadrado. La cualificación y conteo se realizó mediante observación directa y colecta manual; para el caso de los márgenes, se utilizó una red "surber" de 30 x $30 \mathrm{~cm}$, con $0.2 \mathrm{~mm}$ de malla, removiendo el material contenido dentro del 
cuadrante que cubre el lecho. En la zona central del cuerpo hídrico se colectó con una red de cabo y de forma manual a los animales sésiles o fijos a sustratos como troncos caídos. En la búsqueda se procuró abarcar todas las áreas de hospedaje de los organismos, tales como pequeños remansos, hojarasca, piedras, arena, lodos y vegetación riparia.

El material colectado se fijó en alcohol al $70 \%$ y se transportó en bolsas plásticas rotuladas.

El procesamiento de los especímenes se inició depositando la muestra en bandejas plásticas de color blanco para favorecer la separación por el contraste de su color, en algunos casos fue necesario el tamizado y lavado. Finalmente, se conservaron en alcohol al $70 \%$.

La identificación y conteo se llevó a cabo empleando un estereoscopio binocular Olimpus SZX9 con 20X y 40X. Las determinaciones se realizaron con base en las claves propuestas por Hubbard (1990), Flint (1991) la guía para el estudio de macroinvertebrados de Roldán (1996), Merrit y Cummins (2008) y Domínguez y Fernández (2009).

\section{Muestreo físico y químico}

En cada estación muestreada se registraron parámetros físicos del curso de agua:

1. Ancho y profundidad estimada del cuerpo de agua.

2. Claridad del agua, visibilidad o turbidez (observación directa)

3. Velocidad de la corriente, estimada visualmente al arrojar objetos flotantes haciéndolos pasar por dos puntos con una longitud conocida, tomándose el tiempo entre los dos puntos. - Determinación de caudal.

4. Descripción de factores topográficos destacables (básicamente tipo de fondo), y de vegetación en el curso de agua

En cada muestreo se registraron los siguientes parámetros físico-químicos: Temperatura, $\mathrm{pH}$, conductividad, formas de nitrógeno, sólidos suspendidos y totales, alcalinidad, dureza, DQO y Oxígeno Disuelto por el método Winkler.

\section{Fase de análisis}

Datos biológicos

Para establecer el estado ambiental de La quebrada La Cascajosa, se hizo un análisis numérico de forma independiente para cada estación. La composición porcentual media (densidad relativa) de los organismos y los grupos, fue estimada considerando el número de individuos en relación con el total registrado. La diversidad de especies se calculó a través del índice de Shannon - Wiener. Y se determinaron también los índices de Equitatividad y Dominancia de Simpson.

Para facilitar el cálculo de los índices, se construyó una matriz generada por el Programa estadístico Past.
Finalmente, se realizó un estudio de bioindicación con los estándares de calidad biológica del agua, propuestos en el índice de biomonitoreo BMWP (Hellawell, 1989, Roldán, 2001; Zamora, 2007).

\section{Datos físicos y químicos}

La información se organizó en una matriz, en la cual se consignaron los datos obtenidos de cada parámetro físico y químico, en las estaciones de muestreo, para realizar un análisis estadístico descriptivo a nivel espacial y de correlación entre los parámetros evaluados versus estaciones de muestreo.

Análisis de correlación: Se utiliza como una medida del grado de asociación lineal que existe entre dos variables aleatorias. Aleatoria significa para este caso que la variable no está bajo el control del investigador y por lo tanto en su medición puede existir un error asociado (Sierra, 2011). Con el fin de resumir y ordenar la distribución espacio-temporal de macroinvertebrados acuáticos, se empleó un análisis de agrupamiento jerárquico (Clúster), construido a partir de la medida de distancia euclidiana.

\section{Resultados}

\section{Macroinvertebrados}

El grupo de macroinvertebrados acuáticos está constituido fundamentalmente por formas bentónicas que se pueden observar a simple vista; dentro de ésta categoría se incluyen varios grupos entre los cuales los más importantes son los artrópodos, y en particular las larvas de los insectos Sierra (2011).

En términos generales se puede decir que de acuerdo con Segnini (2003), la biota acuática cambia su estructura y funcionamiento al modificarse las condiciones ambientales de sus hábitats naturales, de modo que es posible usar algunas características o propiedades estructurales y funcionales de los diferentes niveles de organización biológica, para evaluar en forma comparativa el estado de la biota acuática, cuya condición es reflejo del estado ecológico del cuerpo de agua.

\section{Composición}

El estado de salud de un río viene dado por la diferencia que existe en la composición de taxa entre los sitios impactados y los de referencia siempre y cuando ambos grupos de sitios tengan condiciones fisicoquímicas similares Norris y Hawkins (2000) citados por Segnini (2003).

En los puntos de muestreo se registró un total de 891 individuos; pertenecientes a 5 clases, 13 órdenes, 29 familias, 33 géneros. En cuanto a número de individuos, el Orden Diptera fue el más representativo con 212; seguido de Ephemeroptera con 178, Trichoptera y Coleoptera con 158 y 115 individuos respectivamente; y de las clases Annelida, Mollusca, Crustacea y 
Platelminta, fueron hallados en menor proporción(15\%).
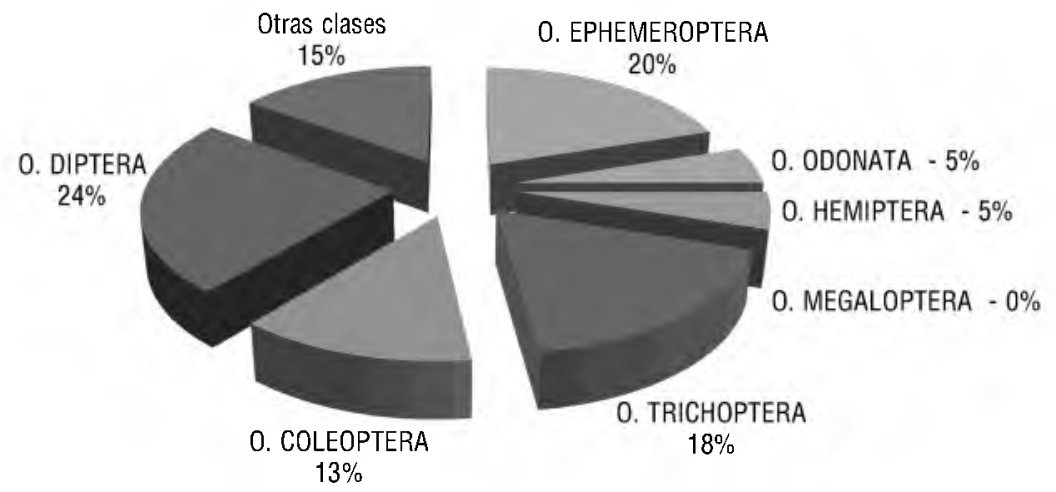

Figura 2. Distribución porcentual de macroinvertebrados acuáticos en La quebrada La Cascajosa, entre noviembre 2011 y mayo 2012.

Con respecto a los resultados registrados para la distribución porcentual de macroinvertebrados acuáticos en el ecosistema en estudio, resultados similares han sido explicados en términos de variabilidad interregional, Jacobsen et al.(2008) soportando en estudios propios y en una amplia gama de investigaciones realizadas por otros autores a nivel mundial, afirma que los cuatro órdenes que constituyen la mayoría de las asociaciones de la corriente de insectos tropicales corresponden a Ephemeroptera, Díptera, Coleoptera y Trichoptera. Este patrón ha sido observado en diversas corrientes africanas (Petrigrove, 1970; Hynes, 1975; Mathooko y Mavuti, 1992; Dobson et al. (2002), citados por (Jacobsen, et al. 2008) y esto parece ser cierto para otras corrientes de áreas tropicales continentales (Jacobsen et al. 2008). Los mismos órdenes dominan la fauna de insectos de la mayoría de los arroyos localizados en zonas de mayor latitud (Hynes, 1970) citado por Jacobsen et al. (2008). Órdenes de insectos Plecoptera, y otros pueden ser localmente abundantes en las corrientes de zonas tropicales, pero, por lo general se producen en cantidades relativamente bajas. Hecho también observado en el ecosistema de la quebrada La Cascajosa.
Las fluctuaciones observadas en cuanto a la distribución. (Figura 3) posiblemente se deben a las variaciones estacionales de los organismos, las cuales se presentan en función del efecto que ocasionan las lluvias o las sequías, que alteran el volumen de agua y modifican la disponibilidad de hábitats. Lo que significa que la variación en los procesos físicos y químicos del agua dada por los cambios climáticos comprometen la sobrevivencia de la comunidad de macroinvertebrados (Posada et al, 2000).

Segnini (2003) afirma que las perturbaciones producen la pérdida o el reemplazo de los taxa que conformaban la biota que originalmente existía en condiciones naturales no degradadas. De manera que la composición de la biota en un sitio impactado debe diferir de la composición de la biota en un sitio en buenas condiciones. Premisa que puede ser comparada en La quebrada La Cascajosa, con el comportamiento de la comunidad de macroinvertebrados acuáticos, en cuanto a su composición a lo largo de las estaciones muestreadas y durante el tiempo de muestreo.

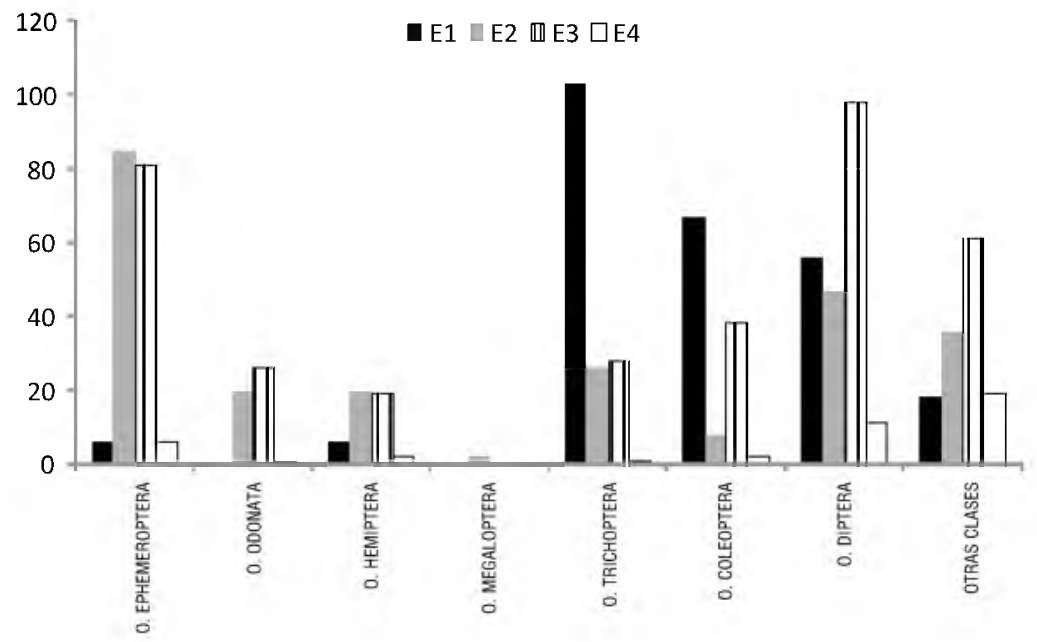

Figura 3. Distribución espacial de macroinvertebrados acuáticos en la quebrada La Cascajosa, entre noviembre 2011 y mayo 2012. 
Según Norris y Hawkins (2000), citados por Segnini (2003), la tasa de pérdida es una medida fundamental de la degradación biológica puesto que los taxa representan las unidades básicas a partir de las cuales se construyen los niveles superiores de organización biológica. Hecho observado en la diferencia de presencia y ausencia de macroinvertebrados en la estación 4 ubicada en la zona urbana y punto de desembocadura del cuerpo de agua. Este sitio fue reconocido como el más alterado, debido a la fuerte intervención antrópica observada, constituida por la disposición inadecuada de basuras en la zona y las descargas de residuos urbanos (domésticos). En esta zona, fueron marcados los cambios en la similitud de la composición de especies. Norris y Hawkins (2000) citados por Segnini (2003) señalan que este tipo de comportamiento, es un indicador de cambios que conllevan riesgo en el ámbito de la comunidad (todas las formas de riqueza) o a nivel del ecosistema (flujo de energía, y ciclo de nutrientes).

Tabla 1. Características de los hábitats de los sitios de muestreo de La quebrada La Cascajosa.

\begin{tabular}{|l|l|l|l|l|}
\hline \multirow{2}{*}{ CARACTERISTICAS } & \multicolumn{4}{|c|}{1} \\
\cline { 2 - 4 } Tipo de sustrato & \multicolumn{1}{|c|}{$\begin{array}{l}\text { Lodo, Materia } \\
\text { orgánica en } \\
\text { descomposición } \\
\text { (hojarasca y } \\
\text { troncos) }\end{array}$} & $\begin{array}{l}\text { Lodo, Materia } \\
\text { orgánica. en } \\
\text { descomposición } \\
\text { (hojarasca, } \\
\text { troncos) }\end{array}$ & $\begin{array}{l}\text { arena, rocas, Materia } \\
\text { orgánica en } \\
\text { descomposición en } \\
\text { algunos sitios } \\
\text { cercanos a las orillas }\end{array}$ & Arena y rocas. \\
\hline Veloc. corriente & lenta & lenta & Moderada & Moderada \\
\hline Color & transparente & transparente & transparente & transparente \\
\hline Cauce & Normal & Normal & Normal & Normal \\
\hline Turbidez & baja & baja & baja & baja \\
\hline Tipo ecosistema & $\begin{array}{l}\text { Bosque húmido } \\
\text { Tropical (Bi-T) }\end{array}$ & $\begin{array}{l}\text { Bosque húmedo } \\
\text { Tropical(Bh-T) }\end{array}$ & Zona urbana & Zona urbana \\
\hline Uso del suelo & $\begin{array}{l}\text { Ganadería, } \\
\text { agricultura de café } \\
\text { y cacao }\end{array}$ & $\begin{array}{l}\text { Ganadería, } \\
\text { agricultura de café }\end{array}$ & Urbanizado & urbanizado \\
\hline Prof. prom. cauce & $5,6 \mathrm{~cm}$. & $13,5 \mathrm{~cm}$. & $28,6 \mathrm{~cm}$. & $36,6 \mathrm{~cm}$. \\
\hline Ancho prom. cauce & $75,2 \mathrm{~cm}$. & $120 \mathrm{~cm}$. & $205 \mathrm{~cm}$. & $420 \mathrm{~cm}$. \\
\hline caudal total & $0,016 \mathrm{~m} / \mathrm{seg}$ & $0,030 \mathrm{~m} / \mathrm{seg}$ & $0,13 \mathrm{~m} / \mathrm{seg}$ & $0,21 \mathrm{~m} / \mathrm{seg}$ \\
\hline Velocidad prom. & $0,075 \mathrm{~m} / \mathrm{seg}$ & $0,040 \mathrm{~m} / \mathrm{seg}$ & $0,052 \mathrm{~m} / \mathrm{seg}$ & $0,047 \mathrm{~m} / \mathrm{seg}$ \\
\hline
\end{tabular}

Tabla 2. Índices ecológicos calculados para la quebrada La Cascajosa.

\begin{tabular}{|c|c|c|c|c|c|c|}
\hline & FECHA & $\begin{array}{l}\text { SHANNON- } \\
\text { WIENNER }\end{array}$ & EQUITATIVIDAD & DOMINANCIA & ABUNDANCIA & RIQUEZA \\
\hline \multirow{5}{*}{ 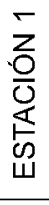 } & nov & 1,76 & 0,80 & 0,2218 & 66 & 9 \\
\hline & dic & 2,04 & 0,93 & 0,1484 & 16 & 9 \\
\hline & feb & 1,59 & 0,82 & 0,2642 & 42 & 7 \\
\hline & marzo & 1,44 & 0,80 & 0,2709 & 33 & 6 \\
\hline & mayo & 1,68 & 0,73 & 0,2617 & 99 & 10 \\
\hline \multirow{5}{*}{ 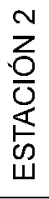 } & nov & 1,86 & 0,89 & 0,1776 & 25 & 8 \\
\hline & dic & 2,05 & 0,89 & 0,1565 & 21 & 10 \\
\hline & feb & 1,61 & 0,89 & 0,2257 & 24 & 6 \\
\hline & marzo & 1,37 & 0,59 & 0,4275 & 66 & 10 \\
\hline & mayo & 1,53 & 0,60 & 0,3739 & 107 & 13 \\
\hline \multirow{5}{*}{ 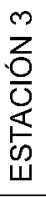 } & nov & 1,51 & 0,68 & 0,2888 & 76 & 9 \\
\hline & dic & 1,75 & 0,76 & 0,2673 & 38 & 10 \\
\hline & feb & 1,61 & 0,90 & 0,2267 & 15 & 6 \\
\hline & marzo & 0,93 & 0,67 & 0,5081 & 108 & 4 \\
\hline & mayo & 1,46 & 0,70 & 0,2958 & 114 & 8 \\
\hline \multirow{5}{*}{ 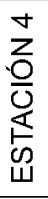 } & nov & 2,03 & 0,88 & 0,172 & 18 & 10 \\
\hline & dic & NR & NR & NR & NR & NR \\
\hline & feb & 0,0 & 0,0 & 0,0 & 0 & 0 \\
\hline & marzo & 0,56 & 0,81 & 0,625 & 4 & 2 \\
\hline & mayo & 1,01 & 0,72 & 0,457 & 19 & 4 \\
\hline
\end{tabular}

^NR: no se realizó muestreo debido a condiciones de orden público que generaron difícil acceso a la zona. 


\section{Abundancia}

La abundancia de especies describe la estructura de las comunidades en términos de la abundancia proporcional de cada especie, es decir, la proporción que representan los individuos de una especie particular respecto al total de individuos de la comunidad (Martella, et al. 2012). De acuerdo con Lampert y Sommer (1997) citados por Segnini (2003) los cambios de los patrones de abundancia y riqueza de especies son una manera de evaluar el impacto de los diferentes tipos de perturbaciones ambientales sobre las comunidades lóticas. La premisa ecológica que soporta el uso de la diversidad para cuantificar el grado de deterioro de los ríos establece que la estabilidad de una comunidad incrementa con su complejidad.

Al comparar la abundancia de organismos en las estaciones de la quebrada La Cascajosa, se encontró similitud entre las estaciones 1 y 2 , en donde se concentró el 28 y $27 \%$ del total de los individuos registrados, se presentó un aumento en el número de individuos en la estación 3 alcanzando un 39,3\% del total de la población, la tendencia a la disminución de organismos se observó en la estación 4 correspondiente a la parte baja de la microcuenca, donde el número de individuos alcanzó tan sólo al 4,6\% del total de macroinvertebrados registrados en el cuerpo de agua durante el tiempo de muestreo.

Ramírez y Viña (1998) afirman que el clima del trópico impide la consolidación de ecosistemas estables y permanentes y por el contrario, se mantienen en constantes procesos de sucesión con proliferación de especies oportunistas cada vez que suceden cambios ambientales. Por tanto, el patrón de la estacionalidad climática, es una influencia en casi todos los aspectos de la ecología de invertebrados de ríos, en particular, de su distribución, las historias de vida y el calendario de eventos del ciclo de vida. Las fluctuaciones climáticas Inter-anuales y perturbaciones tales como las sequías y las tormentas intensas también pueden tener importantes implicaciones para la fauna de macroinvertebrados acuáticos (Jacobsen et al. 2008).

Noviembre (2011) representó el final de temporada seca y las lluvias durante ese mes fueron escasas, en este período se realizó el muestreo 1. Durante los meses de diciembre (2011), y febrero (2012) se presentaron lluvias constantes en la zona, temporada en la que se llevó a cabo los muestreos 2, y 3 y coincide con el menor registro de individuos para las 4 estaciones de muestreo, durante el mes de marzo (2012) las lluvias se presentaron de manera intermitente reduciéndose hacia el mes de abril y mediados del mes de mayo, meses en los que se llevó a cabo los muestreos 4 y 5 .

En la quebrada, las estaciones 1 y 2, presentaron un comportamiento análogo posiblemente debido a su similitud en cuanto a fisiografía y paisaje, ya que las dos estaciones están ubicadas en zona rural. Al analizar la variación de la abundancia de macroinvertebrados, se observó un ligero aumento en todos los sitios evaluados durante los meses en los que la lluvia fue escasa (noviembre 2011, mayo 2012).

\section{Abundancia relativa}

La categoría de abundancia que se presenta en este estudio para cada especie sigue los criterios de Tirira (2007) citado por la Pontificia Universidad Católica de Esmeraldas (2011). De acuerdo con esto, las especies pueden ser comunes, frecuentes, no comunes, raras y de condición desconocida. Es importante aclarar que la abundancia en este caso se refiere a la información que se tenga de cada especie en todo su rango de distribución. Es decir, a diferencia de la abundancia relativa (analizada para los resultados generales y para cada localidad), no considera que tan frecuente fue la observación o captura de cada especie dentro de esta investigación.

Tabla 3. Criterios para estimar la abundancia relativa de las especies. (Tirira, 2007) citado por Pontificia Universidad Católica de Esmeraldas (2011)

\begin{tabular}{|c|c|}
\hline Criterio & Número de individuos por especie \\
\hline Rara & $1-3$ \\
\hline Poco común & $4-10$ \\
\hline Común & $11-25$ \\
\hline Abundante & $>25$ \\
\hline
\end{tabular}

En función de estos parámetros se categoriza a las especies como:

Abundante: Especie muy frecuente y fácil de encontrar Común: Especie encontrada periódicamente aunque en menores densidades.

Poco común: Especie encontrada con poca frecuencia.

Rara: Especie difícil de encontrar y ausente en muchas localidades.

En la quebrada La Cascajosa, durante el período de estudio, como se esquematiza en las Tablas 4 a 7 la frecuencia de aparición de las especies de macroinvertebrados presentó el siguiente comportamiento 
Tabla 4. Frecuencia de aparición de las especies de macroinvertebrados en la quebrada La Cascajosa en la estación 1.

\begin{tabular}{|l|l|l|l|}
\hline Raras 1 -3 & Poco común 4 -10 & Común 11 -25 & abundante $\mathbf{~ 2 5}$ \\
\hline Limnocoris & Leptohyphes & Dugesia sp. & Smicridea \\
\hline Rheumatobates & Chironomini & & Leptonema \\
\hline Mesoveloidea & Limonia & & Anchytarsus \\
\hline Atopsyche & Psichodidae sp1 & & Simulium \\
\hline Stenus & Pseudotelphucidae & & \\
\hline Hidrocanthus & Heterelmis & & \\
\hline Tipuliidae sp1 & & & \\
\hline Hexatoma sp. & & & \\
\hline Scolopendromorfa & & & \\
\hline
\end{tabular}

Tabla 5. Frecuencia de aparición de las especies de macroinvertebrados en la quebrada La Cascajosa en la estación 2.

\begin{tabular}{|l|l|l|l|}
\hline Raras 1 -3 & Poco común 4 -10 & Común 11 -25 & Abundante > 25 \\
\hline Corydalus & Trycoritodes & Leptohyphes & Haplohyphes \\
\hline Anchytarsus & Argia sp. & Hetaerina sp. & Chironomini \\
\hline Derallus & Limnocoris & Rhagovelia sp. & \\
\hline Tubificiidae sp1 & Leptonema sp. & Smicridea & \\
\hline Sphaeridae sp1 & Heterelmis & Physa sp. & \\
\hline & Simulium & & \\
\hline & Dugesia sp. & & \\
\hline & Pseudotelphucidae & & \\
\hline
\end{tabular}

Tabla 6. Frecuencia de aparición de las especies de macroinvertebrados en la quebrada La Cascajosa en la estación 3

\begin{tabular}{|l|l|l|l|}
\hline Raras 1 -3 & Poco común 4 -10 & Común 11 -25 & Abundante > 25 \\
\hline Haplohyphes & Argia sp. & Trycorithodes & Leptohyphes \\
\hline Libelluliinae & Heterelmis & Hetaerina sp. & Leptonema sp. \\
\hline Limnocoris & & Rhagovelia sp. & Macrelmis \\
\hline Limonia & & Chironomini & Simulium \\
\hline Dugesia sp. & & & Physa sp. \\
\hline
\end{tabular}

Tabla 7. Frecuencia de aparición de las especies de macroinvertebrados en la quebrada La Cascajosa en la estación 4.

\begin{tabular}{|l|l|l|l|}
\hline Raras 1 -3 & Poco común 4 -10 & Común 11 -25 & Abundante > 25 \\
\hline Haplohyphes & Leptohyphes & Physa sp. & \\
\hline Hetaerina sp. & Chironomini & & \\
\hline Limnocoris & Dugesia sp. & & \\
\hline Euryguerris & & & \\
\hline Atanatulica & & & \\
\hline Heterelmis & & & \\
\hline Hidrocanthus & & & \\
\hline Tubificidae sp1 & & & \\
\hline Planorbiidae sp1 & & & \\
\hline
\end{tabular}


La información registrada en las tablas $(4 ; 5 ; 6$ y 7$)$ indica que especies bastante tolerantes se encuentran en mayor abundancia relativa especialmente en las estaciones 2 y 3 que por estar influenciadas por actividades agrícolas y urbanas que generan desechos y que finalmente se depositan en estas estaciones, por tanto, tienen cierto nivel de alteración, como ocurre con Smicridea, Leptonema, Anchytarsus, Haplohyphes, Chironomini, Trycorithodes, Physa, los cuales predominan en las dos categorías de común y abundante; aunque no ocurre lo mismo con Simulium que es poco tolerante; es consecuente que Simulium, predomine en la estación 1 por tener mayor velocidad de corriente y menos alteración. Lo anterior obedece a un criterio de número de individuos que puede tener muchas variaciones, y que es muy limitado en la estación 4 probablemente por mayor alteración sobre las poblaciones, ya que en esta última estación las abundancias de todas las especies están muy reducidas, aún para algunas muy tolerantes como Tubificidae o Planorbiidae. En las categorías de rara y poco común se encuentra mayor riqueza de especies pero con menor número de individuos, lo que también concuerda con las diferencias de alteración en las estaciones.

\section{Riqueza (S).}

Se conceptualiza como el número total de especies obtenido por un censo de la comunidad. La riqueza específica (S) es la forma más sencilla de medir la biodiversidad, ya que se basa únicamente en el número de especies presentes, sin tomar en cuenta sus valores de importancia (Martella, et al. 2012)

Como se indica en la Tabla 2 la riqueza de especies en la Estación 1, aumentó, siendo más significativa en el último mes y primer mes de muestreo, coincidiendo con la reducción de las lluvias y la velocidad de la corriente a diferencia de la Estación 2, que presentó similitud en la riqueza durante el periodo de estudio. Cabe destacar que en este punto de muestreo, se presenta el aporte de la quebrada La Cartagena y puede estar contribuyendo a la no reducción en la composición de especies, a pesar del fuerte arrastre que se presenta en el período lluvioso, hecho corroborado durante los muestreos 3 y 4 en los que hubo la mayor precipitación, sin embargo se mantuvo la estabilidad de la riqueza de especies, y de las poblaciones con respecto a las demás estaciones. La Estación 3, tuvo un comportamiento similar a la estación 1 en cuanto al número de los taxones presentándose la menor riqueza durante los meses 3 y 4 (período de lluvias).

La riqueza (S) de macroinvertebrados en la estación 4 fue mayor en el primer muestreo (Noviembre) y presentó una disminución drástica hacia el 2 muestreo (Diciembre) cuando el número de taxones fue 0 debido posiblemente al fuerte arrastre ocasionado por la lluvia presentada horas antes del muestreo. En los muestreos 3 y 4 (marzo y mayo) la riqueza de macroinvertebrados tuvo una ligera tendencia creciente debido a la diferencia en condiciones climáticas, en las que las lluvias fueron intermitentes y ya para el último mes, escasas.

En general, la diferencia en la riqueza para la comunidad de macroinvertebrados, por estaciones de muestreo a lo largo de La quebrada La Cascajosa, se puede explicar desde el punto de vista del gradiente altitudinal y de otros factores como el clima, que contribuyen a la riqueza de especies en los arroyos tropicales además de una mayor disponibilidad de alimentos y diversidad de hábitats (Covich, 1988, citado por Jacobsen et al. 2008) y la intensidad de la depredación (Stout y Vandermeer, 1975; Fox, 1977, citado por Jacobsen et al. 2008)

\section{Diversidad Biológica}

El índice de Diversidad de Shannon - Wiener (1949) tiene establecida una escala que varía entre 0 y 5 bits/individuo, siendo 5 la máxima diversidad; con base en éste rango, se observó que los valores encontrados en el estudio de La quebrada La Cascajosa, alcanzaron el máximo valor en la Estación 2 con 2,05 bits/individuo (mediana diversidad según la escala del índice) (mes de diciembre) y en la estación 1 con 2,04 bits/individuo en el mismo muestreo (mediana diversidad según la escala del índice), seguido por la Estación 4 en el primer mes de muestreo (Noviembre) con 2,03 bits/individuo (mediana diversidad según la escala del índice). Sin embargo en ésta localidad se obtuvieron los mínimos valores en los siguientes muestreos: 0 - 0,5 y 1,0 bits/individuo (poca diversidad según la escala del índice) (tabla 2).

En la Estación 2 se observó variación en la composición de organismos, debido a que el cauce de la quebrada recibe directamente los aportes de la quebrada La Cartagena y aguas abajo en la Estación 3, tiende a estabilizarse tanto la abundancia como la diversidad de especies, siguiendo el mismo patrón de la Estación 1; a través del índice de diversidad de Shannon-Wiener se observó una tendencia a la recuperación del ecosistema demostrando la capacidad de autodepuración y recolonización de organismos bentónicos.

En general, a lo largo del estudio fue la estación 4 la que presentó los valores más bajos. Según Shieh et al (1999), citado por Pave y Marchese (2005), los ríos urbanos están fuertemente influenciados por fuentes puntuales y difusas de contaminación, con contenidos de nutrientes orgánicos e inorgánicos asociados a la urbanización, actividades industriales y agrícolas. Para La estación 4 por estar altamente influenciada por actividades urbanas, se podría hipotetizar que es la más afectada, y donde diferentes tipos de contaminantes (observaciones directas) tienen un efecto sinérgico sobre la comunidad bentónica, simplificando su estructura con una menor riqueza y diversidad de especies a lo largo de los meses muestreados y de las localidades de estudio.

Lo anterior también resulta acorde con el planteamiento de Perkins (1983), citado por Zilli y Gagneten 
(2005) en el que se especifica que todos los factores en los ambientes acuáticos tienen una paridad, salvo sus niveles de polución, por lo cual las localidades más contaminadas deben ser las menos diversas.

\section{Índice de Equitatividad}

En la quebrada La Cascajosa, la equitatividad de la comunidad de macroinvertebrados denotó un patrón similar entre las estaciones de muestreo durante el 2 muestreo con los valores más altos, lo cual significa que la abundancia de organismos estuvo bien distribuida en cada localidad, mientras que en el muestro 4, hubo tendencia a la reducción en los valores de equitatividad.

\section{Índice de Dominancia de Simpson}

Los valores de éste índice oscilan entre 0 y 1 unidades, siendo inversamente proporcionales a la diversidad de especies. En la quebrada La Cascajosa, la dominancia en las comunidades de macroinvertebrados acuáticos estuvo representada por los órdenes Megaloptera y Coleoptera que fueron los órdenes menos diversos pero que por el tamaño de los organismos estuvieron abarcando un mayor espacio. En la tabla 2, se observa que la mayor dominancia, se presentó en las estaciones 3 y 4 correspondientes la zona urbana. Las cuales también obtuvieron los valores más bajos para la diversidad, este hecho, ha sido observado en ambientes con un fuerte grado de perturbación expresado en una alta tasa de extinción y dominancia competitiva (Margalef (1991) Welch (1992) Lampert y Sommer, (1997) Martella et al. 2012).

\section{Índice BMWP (Biological Monitoring Working Party Score System)}

El Biological Monitoring Working Party Score System o Sistema para la Determinación del Índice de Monitoreo Biológico (Índice BMWP), interpretado por Armitage et al. (1983) citado por Zamora (2007), en Gran Bretaña al amparo del "National Water Council", consiste en la ordenación de los macroinvertebrados acuáticos al nivel taxonómico de familia en diez grupos, según una escala de mayor a menor tolerancia a las alteraciones de las condiciones normales naturales de los cuerpos de agua, asignando valores entre uno y diez puntos respectivamente (Zamora, 2007).

Tabla 8. Resultados del índice BMWP para la quebrada La Cascajosa en las estaciones evaluadas

\section{ESTACIÓN 1 : BMWP= 104 = Tipo II aguas limpias. Calidad: Buena}

\begin{tabular}{|c|c|c|}
\hline ORDEN & FAMILIA & PUNTAJE \\
\hline Coleoptera & Ptilodactylidae & 10 \\
\hline Trichoptera & Hydrobiosidae & 9 \\
\hline Hemiptera & Guerridae & \multirow{3}{*}{8} \\
\hline Diptera & Simullidae & \\
\hline Decapoda & Pseudothelpusidae & \\
\hline Ephemeroptera & Leptohyphidae & \multirow{5}{*}{7} \\
\hline Hemiptera & Naucoridae & \\
\hline Trichoptera & Hidropsychidae & \\
\hline Diptera & Psychodidae & \\
\hline Tricladida & Planariidae & \\
\hline Coleoptera & Elmidae & \multirow{2}{*}{6} \\
\hline Coleoptera & Staphylinidae & \\
\hline Hemiptera & Mesoveliidae & 5 \\
\hline Coleoptera & Noteridae & 4 \\
\hline Diptera & Tipulidae & 3 \\
\hline Diptera & Chironomidae & 2 \\
\hline
\end{tabular}

ESTACIÓN 2 . BMWP=91. Tipo III : Aguas medianamente contaminadas. Calidad: Aceptable.

\begin{tabular}{|c|c|c|}
\hline ORDEN & FAMILIA & PUNTAJE \\
\hline Coleoptera & Ptilodactylidae & 10 \\
\hline Hemiptera & Veliidae & \multirow{3}{*}{8} \\
\hline Diptera & Simuliidae & \\
\hline Decapoda & Pseudothelpusidae & \\
\hline Ephemeroptera & Leptohyphidae & \multirow{6}{*}{7} \\
\hline Odonata & Coenagrionidae & \\
\hline Odonata & Calopterygidae & \\
\hline Hemiptera & Naucoridae & \\
\hline Trichoptera & Hydropsychidae & \\
\hline Tricladida & Planariidae & \\
\hline Coleoptera & Elmidae & \multirow{2}{*}{6} \\
\hline Megaloptera & Corydalidae & \\
\hline Bivalvia & Sphaeridae & 4 \\
\hline Bassomatomorpha & Physidae & \multirow[b]{2}{*}{3} \\
\hline Coleoptera & Hydrophilidae & \\
\hline Diptera & Chironomidae & 2 \\
\hline Haplotaxida & Tubificidae & 1 \\
\hline
\end{tabular}


ESTACION 3: BMWP= 78 = Tipo III : Aguas medianamente contaminadas. Calidad: Aceptable.

\begin{tabular}{|c|c|c|}
\hline ORDEN & FAMILIA & PUNTAJE \\
\hline Hemiptera & Veliidae & \multirow[b]{2}{*}{8} \\
\hline Diptera & Simuliidae & \\
\hline Ephemeroptera & Leptohyphidae & \multirow{6}{*}{7} \\
\hline Odonata & Coenagrionidae & \\
\hline Odonata & Calopterygidae & \\
\hline Hemiptera & Naucoridae & \\
\hline Trichoptera & Hydropsychidae & \\
\hline Tricladida & Planariidae & \\
\hline Coleoptera & Elmidae & \multirow{2}{*}{6} \\
\hline Odonata & Libellulidae & \\
\hline Basommatophora & Physidae & \multirow[b]{2}{*}{3} \\
\hline Diptera & Tipulidae & \\
\hline Diptera & Chironomidae & 2 \\
\hline
\end{tabular}

ESTACION 4: BMWP = 65 = Tipo III aceptable: aguas medianamente contaminadas

\begin{tabular}{|l|l|c|}
\hline ORDEN & FAMILIA & PUNTAJE \\
\hline Trichoptera & Leptoceridae & \multirow{2}{*}{8} \\
\cline { 1 - 2 } Hemiptera & Guerridae & \\
\cline { 1 - 2 } Ephemeroptera & Leptohyphidae & \multirow{2}{*}{7} \\
\cline { 1 - 2 } Odonata & Calopterygidae & \multirow{2}{*}{7} \\
\cline { 1 - 2 } Hemiptera & Naucoridae & \\
\hline Tricladida & Planariidae & 6 \\
\hline Coleoptera & Elmidae & 5 \\
\hline Bassomatomorpha & Planorbiidae & 4 \\
\hline Coleoptera & Noteridae & 3 \\
\hline Bassomatomorpha & Physidae & 2 \\
\hline Diptera & Chironomidae & 2 \\
\hline Haplotaxida & Tubificidae & 1 \\
\hline
\end{tabular}

De acuerdo con el BMWP, La estación I tiene aguas clase II buena (aguas limpias), las demás estaciones presentan agua Clase III, aceptable (ligeramente contaminada); sin embargo, E4 presentó el valor más bajo para aguas Clase III, (aguas moderadamente contaminadas). Con los valores del BMWP se construyó el mapa de calidad de agua de la quebrada y se relaciona con las fuentes de contaminación del agua, se nota que la estación E4 es la que tiene aguas de más baja calidad, porque recibe las aguas de E3 por estar ubicada en plena zona urbana en donde la descarga de residuos domésticos es constante e incontrolada, por lo tanto ésta es la estación más expuesta a las fuentes de contaminación.

Las estaciones de muestreo forman un gran grupo en el cual se diferencian dos subdivisiones importantes. La primera subdivisión (E 1 y E 2) estuvo formada por las estaciones menos intervenidas, situadas en la parte alta de La quebrada. La otra subdivisión comprendió las estaciones ubicadas en la parte media y baja de la micro-cuenca (E3, E4) zona urbana; dentro de esta subdivisión las que presentaron mayor puntaje para el índice fue E1 que por su puntaje se acerca a aguas tipo I mientras que la estación $\mathrm{E} 4$, la que recibe la mayor cantidad de descargas de aguas residuales en relación con las demás estaciones, se separó del resto obteniendo el más bajo puntaje para el índice.

En la quebrada La Cascajosa, la pendiente del canal hace que estas aguas sean rápidas y que el lecho permanezca lavado sin mucha acumulación de material finamente particulado. En la estación 3 y 4 el sustrato del lecho está conformado por rocas de gran tamaño que influyen en la turbulencia del agua y, por consiguiente, en el incremento de la concentración de oxígeno, factor importante para la recuperación de la calidad del agua, porque se incrementa la capacidad oxidante de las quebrada para mineralizar los aportes de materia orgánica que llegan a su cauce. Debido a esto es muy probable que los resultados para el índice BMWP en las estaciones muestreadas no se registre calidad de agua tipo dudosa o crítica a pesar de las observaciones directas que se hace en el recorrido frente a la extrema contaminación causada por los desechos originados por las actividades domésticas (aguas residuales, residuos sólidos).

Es muy probable que en esta quebrada las características del lecho sean determinantes en la estructura 
de la comunidad de macroinvertebrados acuáticos. El sustrato del lecho conformado por lodo y hojarasca en la estación I, arena y rocas de diferente tamaño más que todo medianas y grandes, a partir de la estación II hasta la IV, posibilita el movimiento del agua a lo largo del cauce y constituye una base firme para la fijación o adherencia de organismos de vida sedentaria. Estas características al ser combinadas con la pendiente a lo largo de La quebrada, propician una alta diversidad de hábitats para los macroinvertebrados, factores que inciden en la mediana biodiversidad de especies registradas.

El caudal y el área transversal tienen tendencia a aumentar hacia su desembocadura E4. Sin embargo, se observó que a pesar de que La quebrada se crece con las lluvias fuertes y hay un arrastre de materiales y macroinvertebrados, el número de taxa y de individuos encontrados en la mayoría de las estaciones durante los cinco muestreos presentaron variaciones espaciales y temporales importantes pero no tan marcadas, por estación, lo cual puede estar relacionado con la homogeneidad del sustrato en la mayoría de las estaciones.

Las estaciones II, III y IV están afectadas principalmente por la contaminación con escorrentías de cultivos, residuos sólidos y vertimientos de aguas residuales domésticas y pecuarias. Esta quebrada, aguas abajo de la estación E2, presenta un mayor número de fuentes de contaminación por estar ubicada en el área urbana del municipio de Garzón. La estaciones de La quebrada registraron valores de oxígeno que posibilitan el desarrollo de la vida, este factor es concluyente también para justificar los valores registrados por el índice BMWP.

\section{Parámetros físicos y químicos}

Con relación a las variables físicas y químicas, en cada muestreo se registró temperatura, $\mathrm{pH}$, conductividad, formas de nitrógeno, sólidos suspendidos y totales, alcalinidad, dureza, oxígeno disuelto y DQO por el método Winkler. Los parámetros medidos se referencian en la Tabla 12, con los valores promedio para cada estación de muestreo.

Tabla 9. Parámetros físicos y químicos registrados en la quebrada La Cascajosa.

\begin{tabular}{|c|c|c|c|c|c|}
\hline PARÁMETRO & UNIDADES & QUEB & $\begin{array}{l}\text { ADA L } \\
\text { or prc } \\
\text { medi }\end{array}$ & $\begin{array}{l}\text { CASC } \\
\text { edio d } \\
\text { nes) }\end{array}$ & $\begin{array}{l}\text { JOSA } \\
5\end{array}$ \\
\hline \multirow[b]{2}{*}{ Tempe ratura } & \multirow[b]{2}{*}{${ }^{\circ} \mathrm{C}$} & E1 & E2 & E3 & E 4 \\
\hline & & 20,14 & 22,7 & 23,8 & 22,4 \\
\hline $\mathrm{pH}$ & Unidades & 7,5 & 8 & 7,2 & 6,9 \\
\hline Conductividad & $\mu S / c m$ & 6,7 & 9,8 & 18 & 12 \\
\hline Sólidos totales & $\mathrm{mg} / \mathrm{l}$ & 3,2 & 6,3 & 66 & 44,6 \\
\hline Oxígeno disuelto & $\mathrm{mgO}_{2} \mathrm{~L}^{-1}$ & 8,8 & 8,2 & 8,1 & 8 \\
\hline Demanda Química de oxigeno DQO. & $\mathrm{mgO}_{2} \mathrm{~L}^{-1}$ & 65 & 80 & 80 & 83 \\
\hline Nitrógeno & $\mathrm{mg} / \mathrm{l}$ & 6,1 & 6,1 & 6 & 5,32 \\
\hline Nitrógeno amoniacal(NH3-N) & $\mathrm{mg} / \mathrm{l}$ & 0,02 & 0,1 & 0,2 & 0,4 \\
\hline Nitritos(NO2-N) & $\mathrm{mg} / \mathrm{l}$ & 0,05 & 0,05 & 0,06 & 0,06 \\
\hline Alcalinidad & $\mathrm{mmol} \mathrm{CaCO}_{3} / \mathrm{L}$ & 0,2 & 0,8 & 0,2 & 0,2 \\
\hline Dureza & $\mathrm{mgCaCO}_{3} / \mathrm{l}$ & 25 & 25 & 30 & 46 \\
\hline
\end{tabular}

\section{Temperatura}

La probabilidad de encontrar en la naturaleza aguas que mantengan su temperatura constante durante todo el año es muy escasa (Rivera et al. 2004), aunque en los cuerpos de agua tropicales se presenta como característica fundamental, una temperatura más o menos uniforme a lo largo de todo el año (Roldán, 1992).

En la quebrada La Cascajosa, las temperaturas registradas en las diferentes estaciones de muestreo, durante el período de estudio, fluctuaron entre 20,1 y $24,5^{\circ} \mathrm{C}$ (Tabla 9 éste parámetro se considera influyente para el mantenimiento de la vida acuática, el flujo de aguas corrientes, no debe aumentar en su valor natural en más de $3^{\circ} \mathrm{C}$ llegando a tolerar hasta un máximo de $30^{\circ} \mathrm{C}$ (Rivera y Muñoz 1999). De acuerdo con los datos de la Tabla $9, \mathrm{y}$ con base en lo anterior, el agua de la quebrada La Cascajosa, cumple con los requisitos mínimos en cuanto a temperatura para conservar la vida acuática y consecuentemente su tolerancia. 
Los valores máximos se registraron en mayo (muestreo 5), época en la que se presentaron muy pocas lluvias y la radiación solar tendió a ser mayor que en las demás épocas de muestreo. La estación que registró los más altos valores fue la 3 (la más "urbana") en el muestreo del mes de mayo; y las menores ocurrieron en la estación 1 en diciembre y febrero; Estos valores mínimos contribuyeron para que en esta localidad el oxígeno disuelto fuera un poco mayor (Tabla 9), ya que la temperatura es una variable de gran importancia en la disolución de este gas (Roldán, 1999). Tal condición pudo contribuir a que en la estación 1 y en la estación 2 se registraran los mayores valores para la diversidad (Tabla 2).

En general las fluctuaciones de temperatura del agua fueron notorias pero no abruptas (al menos para las horas en que se hicieron las mediciones). Esta es una característica de los ecosistemas tropicales donde las temperaturas no sufren grandes variaciones a lo largo del año, como las que ocurren en las zonas templadas debido a los cambios estaciónales (Roldán et al. 2001). Para Jacobsen et al. (2008) el desarrollo de la fauna de los insectos y otros macroinvertebrados tropicales es afectado por la temperatura, pero el régimen de flujo y la estabilidad del hábitat tiene una influencia primaria en los parámetros del ciclo vital y dinámica de la población.

\section{Potencial de Hidrógeno $(\mathrm{pH})$}

El pH del agua representa su acidez o basicidad, cuyo factor más importante es habitualmente la concentración en anhídrido carbónico debida a la mineralización total (Rodier, 1990), éste parámetro depende de la geoquímica del suelo, de la dinámica física y química del bióxido de carbono, ácido carbónico, carbonatos, bicarbonatos, ácidos húmicos y fúlvicos.

En las estaciones muestreadas de la quebrada La Cascajosa, el pH varió entre 6,8 y 8,1 unidades, presentando el valor más bajo en la Estación 4 y el más alto en la estación 2. En general las condiciones fueron cercanas a la neutralidad para la mayoría de las estaciones. Los valores registrados no son limitantes para la vida, pues el $\mathrm{pH}$ no debe ser menor a $4.5 \mathrm{ni}$ mayor a 8.5, valores límites para la supervivencia de organismos acuáticos (Machado y Roldán, 1981). Otros autores como Flanagan (1992) citado por Rivera (2011), plantean que el ámbito normal en el cual pueden fluctuar los valores de $\mathrm{pH}$ es de 6.5 a 8.0, rango en el cual se encuentran la mayor parte de las estaciones del presente estudio.

El pH en los cuerpos de agua debe mantenerse por encima de 6 para que el mayor porcentaje de nitrógeno durante el proceso de desnitrificación no sea liberado en forma de óxido nitroso y, con ello disminuir el efecto invernadero (Silva, 2002) se puede observar la tendencia de las dos estaciones urbanas (3 y 4) a presentar menores valores del $\mathrm{pH}$, indicando mayor acidez en la estación 4. Lo anterior, se deba posiblemente a la mayor descarga de contaminantes en esta zona, lo que indica mantener el pH en condiciones ligeramente ácidas a través de los períodos muestreados.

\section{Conductividad.}

La conductividad es una medida que permite evaluar aproximadamente la mineralización global del agua (Rodier, 1990), como también la carga iónica presente (Rivera et al. 2004). Los valores encontrados en el estudio fluctuaron entre 6,1 y $20 \mu \mathrm{S} / \mathrm{cm}$ en las diferentes estaciones de muestreo, presentándose la mayor conductividad $(20 \mu \mathrm{S} / \mathrm{cm})$ en la Estación 3 y el menor valor $(6,1 \mu \mathrm{S} / \mathrm{cm}$.) en la Estación 1 de la quebrada La Cascajosa.

De acuerdo con Roldán (1992), los ríos son oligotróficos en su nacimiento, pero su conductividad va aumentando progresivamente a lo largo del cauce por efectos de la erosión, arrastre de sedimento y escorrentía provocada por lluvias.

Con relación a lo anterior, la Estación 2 es influenciada por el aporte de iones a través de la quebrada tributaria La Cartagena, pues en esta estación es un punto de encuentro, y se incrementa su valor promedio (de 6,7 $\mu \mathrm{S} / \mathrm{cm}$ en la Estación 1 a $9,8 \mu \mathrm{S} / \mathrm{cm}$ en la Estación 2), sin embargo, en la Estación 4 la conductividad disminuyó respecto a la estación 3 (donde se registran los valores más altos), debido a la posible autodepuración en su recorrido por la microcuenca (Tabla 9).

Teniendo en cuenta, que en las estaciones de muestreo en La quebrada La Cascajosa, la conductividad presentó valores inferiores a $100 \mu \mathrm{S} / \mathrm{cm}$ durante todo el período de muestreo (Tabla 9) y con base en la escala para evaluación de mineralización de aguas propuesta por Rodier (1990), se puede considerar a La quebrada como un ambiente con baja concentración de iones y de muy débil mineralización; a continuación se detalla este planteamiento:

Escala propuesta por Rodier 1990:

$\begin{array}{ll}\text { Conductividad } & \text { Clasificación } \\ <100 \mu \mathrm{S} / \mathrm{cm} & \text { Mineralización muy débil } \\ <200 \mathrm{HS} / \mathrm{cm} & \text { Mineralización débil } \\ >1000 \mu \mathrm{S} / \mathrm{cm} & \text { Mineralización excesiva }\end{array}$

Las concentraciones más altas se registraron para todas las estaciones en los meses de noviembre y mayo, épocas en las que previo a los muestreos no llovió en la zona, según Chalarca et al. (2007) se deben a la presencia de iones, aniones y, que se concentran por efecto de la evaporación y la disminución de las lluvias. Además, los procesos de degradación de la Materia orgánica aceleran la concentración de iones en el agua. Martínez (2009) señala que los valores altos de conductividad son un buen indicador de condiciones adecuadas para una producción biológica elevada, ésta afirmación podría tener relación con que en la estación 3 donde se registra el mayor valor, también se registró el mayor porcentaje de abundancia para la población 
total de macroinvertebrados registrados durante la época de muestreos en la quebrada La Cascajosa.

\section{Sólidos Totales (ST $\mathrm{mg} \mathrm{L}^{-1}$ )}

Los sólidos totales están compuestos de material sedimentable, en suspensión y diluidos, los cuales se expresan en $\mathrm{mg} \mathrm{L}^{-1}$. En general, este tipo de partículas incrementa los valores de contaminación orgánica e inorgánica en las aguas (Escuela de Ingeniería de Recursos Naturales y del Ambiente, 2001).

En La quebrada, el contenido de sólidos totales, tuvo una tendencia al aumento en su paso a través de la microcuenca, pasando de $3,2 \mathrm{mg} \mathrm{L}^{-1}$ en la parte alta a 66 $\mathrm{mg} \mathrm{L}^{-1}$ en la estación 3 pero se reduce a 44,6 en la estación 4.

Este comportamiento posiblemente se debe a la diferencia altitudinal entre las localidades 1 a 3 y 3 y 4 (de 1124 a $800 \mathrm{msnm}$ ), y la disminución de la velocidad de la corriente en la Estaciones 3 y 4 . Además de la sedimentación natural, en su transcurso, crecen macrófitas acuáticas y gramíneas, que pueden de alguna forma incidir en el comportamiento de los sólidos a lo largo de la microcuenca.

En las Estaciones 1, 2, y 4 los sólidos no registraron diferencias entre meses, (Tabla 4) lo que indica que las condiciones climáticas tuvieron poco efecto sobre las partículas suspendidas. Se ha demostrado que éste parámetro está directamente relacionado con la dinámica de las corrientes en los cuerpos de agua (Metcalf y Eddy 1996) y la corriente de la quebrada La Cascajosa es baja a lo largo de su cauce pero incide en su variación la cantidad de descargas residuales urbanas que ocurren, la diferencia es notable desde la estación 3 a 4 (zonas urbanas) por tanto, se mantiene una fuerte interacción con los componentes abióticos y bióticos del ecosistema.

\section{Oxígeno Disuelto $\left(\mathrm{mgO}_{2} \mathrm{~L}^{-1}\right)$}

El oxígeno disuelto en los ecosistemas lóticos depende de la presión atmosférica, de la temperatura y de la altura sobre el nivel del mar (Catalán, 1969), citado por Guerrero et al. (2003), también depende de las características del cauce, la turbulencia del agua y de los procesos químicos y biológicos (Machado y Roldán, 1981) y es relevante en el control de la calidad de las aguas, siendo su presencia y concentración esencial para sustentar las formas superiores de vida, como también para evaluar los efectos de agentes contaminantes (Rivera et al. 2004).

En la quebrada La Cascajosa el Oxígeno disuelto varió entre 7.6 y $9 \mathrm{mgO}_{2} \mathrm{~L}^{-1}$ (Tabla 4). Durante la época de febrero se registró un promedio mayor de oxígeno y en noviembre y mayo los valores fueron menores.

De acuerdo con Rivera (2011) las fluctuaciones en la concentración de oxígeno están influenciadas por la interacción con la atmósfera y por los procesos fotosintéticos y respiratorios que se producen en el agua. En los meses de Noviembre de 2011 y Mayo de 2012 en la zona se registraron escasas lluvias mientras que para el mes de Febrero las lluvias fueron constantes.

La estación con menores concentraciones fue la 4 debido a los altos valores en la DQO en este sitio. Esto indica, que en esta localidad existe un consumo de este gas posiblemente en reacciones de biodegradación. Según Pérez y Rodríguez (2008) cuando los niveles de oxígeno disuelto son reducidos, el consumo de oxígeno en los procesos de degradación de la materia orgánica agota dicho gas y las condiciones anóxicas que se generan son inapropiadas para la mayoría de los invertebrados. La presencia o ausencia de oxígeno determina qué tipo de microorganismos (aerobios o anaerobios) son los dominantes y por lo tanto influye en la capacidad del agua para llevar a cabo procesos de auto purificación en el ecosistema acuático. En relación con esto, se puede afirmar que la menor diversidad de organismos registrados estuvo en la estación 4 donde estuvieron las menores concentraciones de oxígeno, mientras que para la estación 1 fue la mayor diversidad seguida de la estación 2.

Teniendo en cuenta el Decreto 1594 (Ministerio de Salud República de Colombia, 1984) la concentración más baja de oxígeno disuelto aceptada para la preservación de la vida acuática es de $4 \mathrm{mg} / \mathrm{L}$. Los datos muestran que en la quebrada La Cascajosa los valores registrados para ésta variable, se mantuvieron durante el período de estudio entre los niveles de aceptabilidad (Tabla 4 ).

Los niveles de oxígeno disuelto registrados fueron inversamente proporcionales a la temperatura en las estaciones permaneciendo en unos niveles de saturación aptos para los procesos metabólicos aeróbicos.

\section{Demanda Química de Oxigeno $\mathrm{DQO}(\mathrm{mg} / \mathrm{L})$}

Corresponde a la cantidad de oxígeno necesario para oxidar químicamente, la materia orgánica presente en el agua (Texeira, 1993).

En la estación 1 de la quebrada, se registró un valor $65 \mathrm{mg} / \mathrm{l}$; aguas abajo de esta localidad (Estaciones 2 y 3), se evidenció un incremento en la DQO hasta $80 \mathrm{mg} / \mathrm{l}$, probablemente por los aportes de la quebrada La Cartagena que están incidiendo sobre el cauce de la quebrada.

El comportamiento de la DQO, es inversamente proporcional a los valores obtenidos para el oxígeno disuelto (Ramírez y Viña 1998). Esta tendencia se ajusta directamente a la zona en donde hay incremento de DQO y disminuye el oxígeno disuelto, por la oxidación de la materia orgánica. 


\section{Compuestos Nitrogenados $\left(\mathrm{NH}-\mathrm{N}, \mathrm{NO}_{2}-\mathrm{N}\right.$ y nitrógeno Kjeldahl N)}

De acuerdo con los resultados obtenidos, la cantidad de nitrógeno transportada por el cauce de la quebrada, es similar en todas las estaciones, según Blanco (2002), la materia que entra a un cauce posiblemente recorre tramos cortos río abajo o es atrapada por barreras naturales. Triska et al. (1993), afirma que el transporte y variación de nitrógeno inorgánico puede variar tan solo, en unos pocos metros.

El nitrógeno amoniacal registró valores promedio que van desde 0.02 hasta $0,4 \mathrm{mg} / \mathrm{L}\left(\mathrm{NH}_{3}\right)$. La estación con los mínimos valores fue la 1. La estación con máximos valores fueron la 4 . Los nitritos registraron una fluctuación entre 0.05 y $0.06 \mathrm{mg} / \mathrm{L}$ de $\mathrm{NO}_{2}$. Los máximos valores se presentaron en la estación 3 y en la estación 4. De acuerdo con Roldán (1992) los niveles aceptables de nitritos no deben superar $0.2 \mathrm{mg} / \mathrm{L} \mathrm{y}$ en la quebrada La Cascajosa no se superó este valor. Kadlec y Knight (2001) reportan que los niveles detectables de nitrito en humedales indican asimilación incompleta de nitrógeno y presencia de una fuente de ingreso de origen antrópico. Margalef (1991) indica que la presencia de cantidades altas de nitritos es una evidencia de inestabilidad ambiental del ecosistema acuático, en el caso del ecosistema estudiado se puede observar que hay un aumento de nutrientes a través de las estaciones rurales a urbanas.

En la quebrada, el comportamiento del nitrógeno permaneció estable; este hecho pudo estar influenciado por el tipo de cauce y la baja cantidad de nutrientes en las formas del nitrógeno evaluada en las muestras analizadas, y relacionada con la rápida fragmentación mecánica de la corriente ya que según Triska et al. (1993), es altamente probable que si existe una baja cantidad de detritos a través de los márgenes del río, esta puede ser rápidamente fragmentada por la corriente en tramos cortos; durante el periodo de estudio hubo una variación en la precipitación y la temperatura del aire, con respecto a esto se ha establecido que éstos factores en las cuencas hidrográficas, tienen efecto sobre la vegetación, el suelo y el agua, los cuales de manera conjunta se reflejan en los ciclos de nutrientes (Clair y Ehrman,1996).

Según Blanco (2002) el nitrógeno que ingresa al cauce es procesado rápidamente por algas y microorganismos. Posiblemente este factor también incide en la quebrada La Cascajosa.

\section{Alcalinidad}

Este parámetro es importante, por estar relacionado con la estabilidad del pH del agua (Rivera et al. 2004) y depende en gran parte de la naturaleza del terreno y de las rocas con que está en contacto (Roldán, 1992), según este autor, la alcalinidad es una medida de la cantidad de iones bicarbonato y carbonato presentes en el agua por lo tanto, es una manera de conocer el funcionamiento y metabolismo de un ecosistema acuático.

En la quebrada La Cascajosa, los valores registrados para la alcalinidad fluctuaron entre 0,2 y $0,8 \mathrm{mmol}$ $\mathrm{CaCO}_{3} / \mathrm{L}$, alcanzando el mayor valor en la Estación 2 (Tabla 4). Con base en el planteamiento de Rodier (1990), se establece que una alcalinidad de hasta 150 mg de $\mathrm{CaCO}_{3} / \mathrm{l}$, indica que las aguas son de buena calidad, sugiriendo de esta forma, que los valores para la alcalinidad hallados indican, que la quebrada es un ecosistema estable posiblemente influenciado por las bajas variaciones de $\mathrm{pH}$.

El aumento de la alcalinidad en la Estación 2, está relacionada directamente con un ligero aumento de $\mathrm{pH}$ en esta estación con respecto a las estaciones 1,3 y 4 de la quebrada La Cascajosa.

\section{Dureza Cálcica (mg de $\left.\mathrm{CaCO}_{3} / \mathrm{l}\right)$}

Para las estaciones muestreadas en la quebrada La Cascajosa, la dureza presentó un valor promedio que fluctuó entre en 25 y $46 \mathrm{mg}$ de $\mathrm{CaCO}_{3} / \mathrm{l}$ el mayor valor fue registrado en la estación 4 . El comportamiento de éste parámetro a lo largo de la quebrada, puede estar relacionado con la presencia de materia vegetal a lo largo del cuerpo de agua, ya que según Rodier (1990), en ciertas descargas, la descomposición de la materia vegetal puede conducir a la liberación de anhídrido carbónico, que arrastrado puede disolver el calcio del suelo y contribuir a las variaciones de la dureza.

En cuanto a productividad, según Ohle (1934), citado por Roldán (1992), los valores de la dureza superiores a $25 \mathrm{mg} / \mathrm{L}$ indican un estado medio y altamente productivo, de acuerdo con lo anterior, la quebrada La Cascajosa es medianamente productiva aumentando su producción en la estación 4 .

De acuerdo con Rodier (1990), las aguas de buena calidad soportan hasta $150 \mathrm{mg}$ de $\mathrm{CaCO}_{3} / \mathrm{l}$, las de calidad media hasta $300 \mathrm{mg}$ de $\mathrm{CaCO}_{3}$, las de calidad aceptable y difícilmente utilizables llegan hasta un nivel de $500 \mathrm{mg} \mathrm{de} \mathrm{CaCO}_{3}$, y valores mayores de $600 \mathrm{mg}$ de $\mathrm{CaCO}_{3}$ respectivamente. Según esta clasificación, las aguas de las estaciones evaluadas se incluyen dentro del rango de aguas de un estado medio de productividad.

\section{Análisis de correlación por componentes principales y análisis de clúster.}

El análisis de componentes principales muestra que las variables físicas y químicas nitrógeno, $\mathrm{pH}$ y alcalinidad evaluadas en las estaciones de muestreo inciden de manera significativa en los índices biológicos abundancia, diversidad y riqueza de especies mostrando una amplia correlación entre variables (Figura 4) 


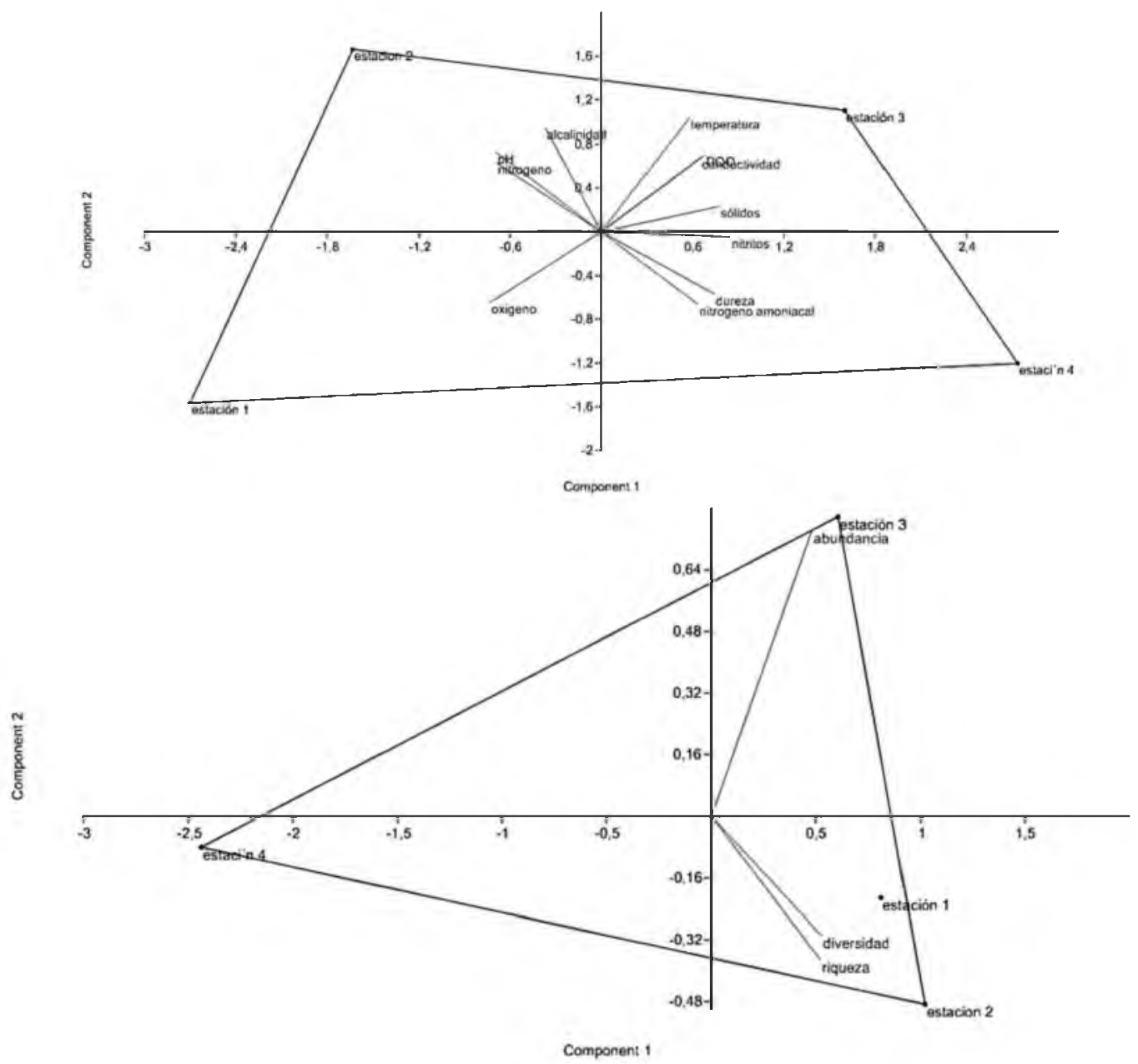

Figura 4. Correlación de las Variables físicas-químicas y biológicas evaluadas en las estaciones de muestreo en la quebrada La Cascajosa.

Según la Figura 4, las estaciones 1, 2 y 3 se encuentran más correlacionadas en cuanto a la abundancia, diversidad y riqueza mostrando mayor porcentaje de similitud, la estación 1 y 2 para la diversidad y riqueza de especies. Estas dos estaciones resultan ser similares (Figura5) pues se encontró una fauna de macroinvertebrados rica debido a la calidad del agua y por estar ubicadas en zona rural a una amplia gama de biotopos que ofrece La quebrada en estas dos estaciones, lo que permite desarrollar las asociaciones de macroinvertebrados en la zona alta de la quebrada La Cascajosa porque la calidad del agua, la disponibilidad de alimento y las características del sustrato, son los principales factores que gobiernan la abundancia y distribución de macroinvertebrados acuáticos (Merrit y Cummins, 2008).

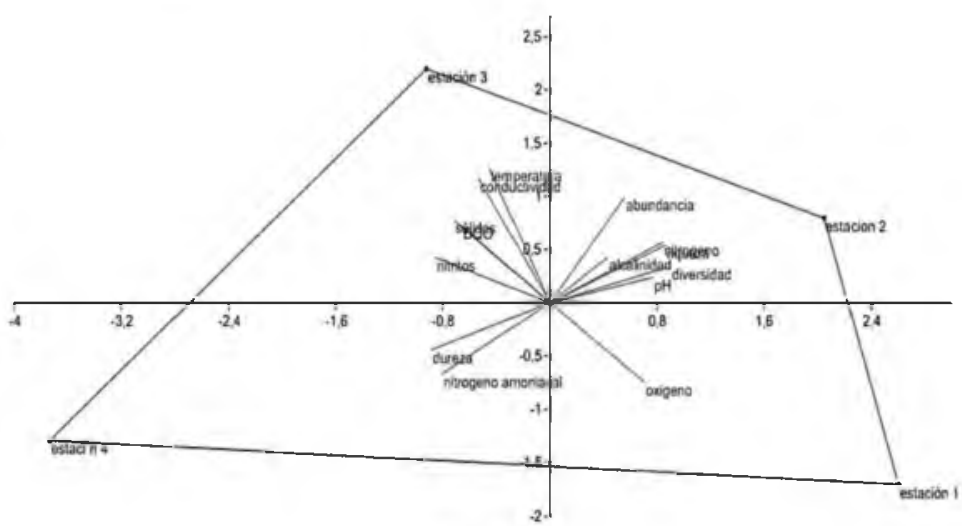

Comporens :

\begin{tabular}{|l|l|l|l|}
\hline & Axis 1 & Axis 2 & Axis 3 \\
\hline estación 1 & 2,6023 & $-1,7016$ & $-1,1093$ \\
\hline estación 2 & 2,0524 & 0,79497 & 1,8021 \\
\hline estación 3 & $-0,9207$ & 2,1974 & $-1,2082$ \\
\hline estación 4 & $-3,734$ & $-1,2908$ & 0,51536 \\
\hline
\end{tabular}

Figura 5. Análisis Multivariable de Co-inercia para las variables muestreadas. 
El Análisis Multivariable de Co-inercia, muestra que la información está principalmente resumida por tres ejes. El primero, explica un 61,3\% de la Variabilidad, el segundo un $23,8 \%$ y el tercero un $14,7 \%$ con una correlación de $r=0.99$. No se evidenció cierta dependencia a las variables ambientales estudiadas y sus poblaciones persisten en el espacio y tiempo en el tipo de hábitat estudiado. Los rasgos biológicos que presentan las poblaciones de invertebrados acuáticos, les confiere cierta resistencia y resiliencia frente a eventos de perturbación (Townsend y Hildrew, 1994 citados por Montoya, 2008), posibilitando su sobrevivencia y posterior persistencia en los hábitat que se desarrollan.

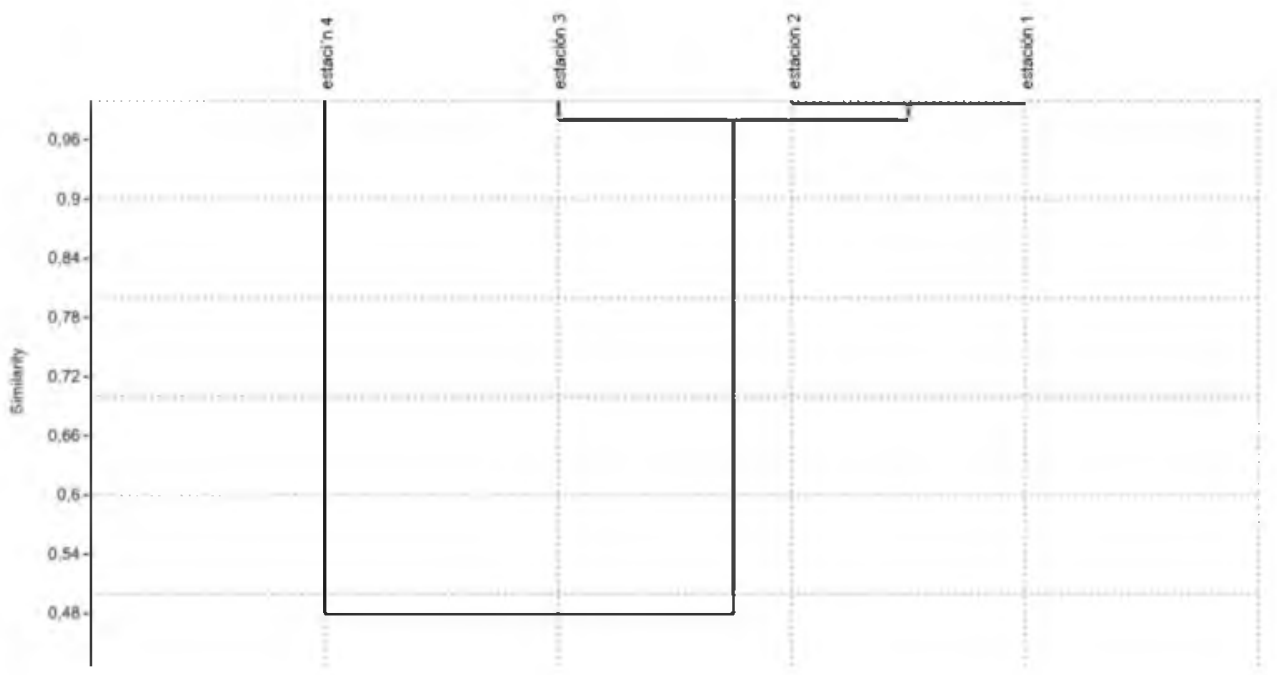

Figura 6. Clúster de Similiridad para las estaciones de muestreo en la quebrada La Cascajosa.

El Clúster de la Figura 6. indica que los sitios que presentaron mayor similaridad fueron las estaciones 1 y 2 con un rango de similaridad por encima del $96 \%$, debido a que el cauce de la quebrada y sus márgenes muestran características homogéneas, y los sitios que presentaron una similaridad diferente la estación 4 con $48 \%$ este sitio presenta esta similitud debido a que está ubicado en la zona urbana y está sometido a agentes contaminantes.
A lo largo de todo el estudio en el clúster de similitudes se muestra diferencias en el comportamiento de asociación de macroinvertebrados acuáticos (Figura 7) para una misma estación y entre las estaciones a lo largo del periodo muestreado lo que puede ser indicador de una dinámica alta para un ambiente pequeño, porque la riqueza numérica de taxones presentó diferencias en porcentajes hasta del $40 \%$ lo que puede estar asociado a pequeñas fluctuaciones del caudal y los ciclos de recolonización de biotopos (Montoya 2008)

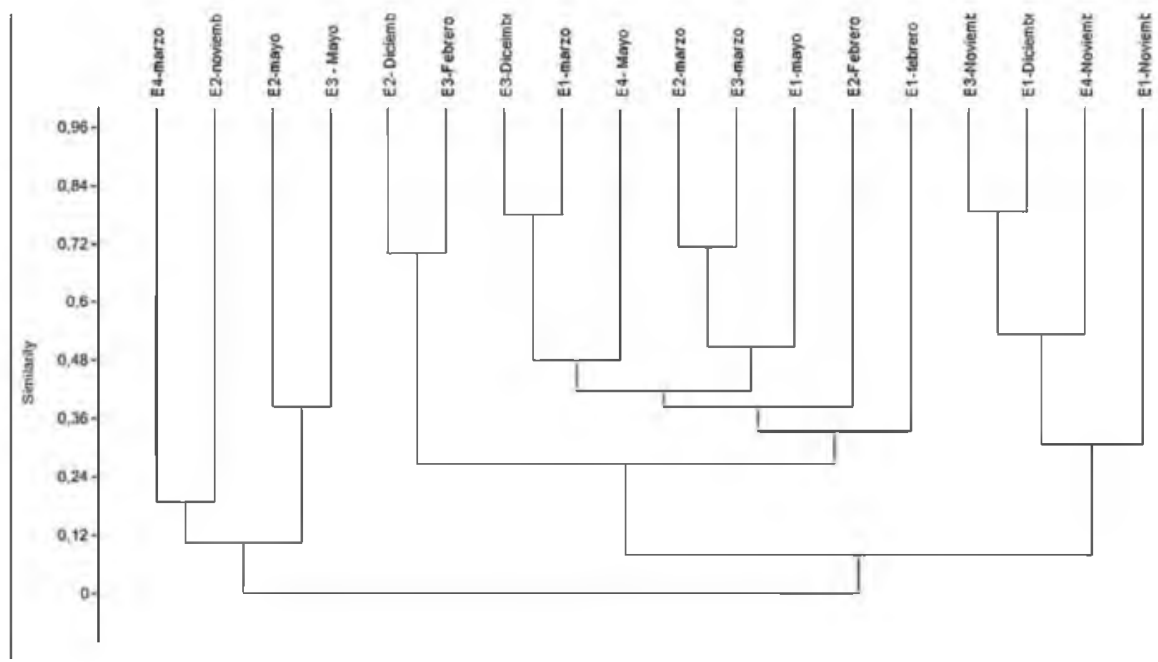

Figura 7. Clúster de similitudes en el comportamiento de asociación de macroinvertebrados acuáticos en la quebrada La Cascajosa. 


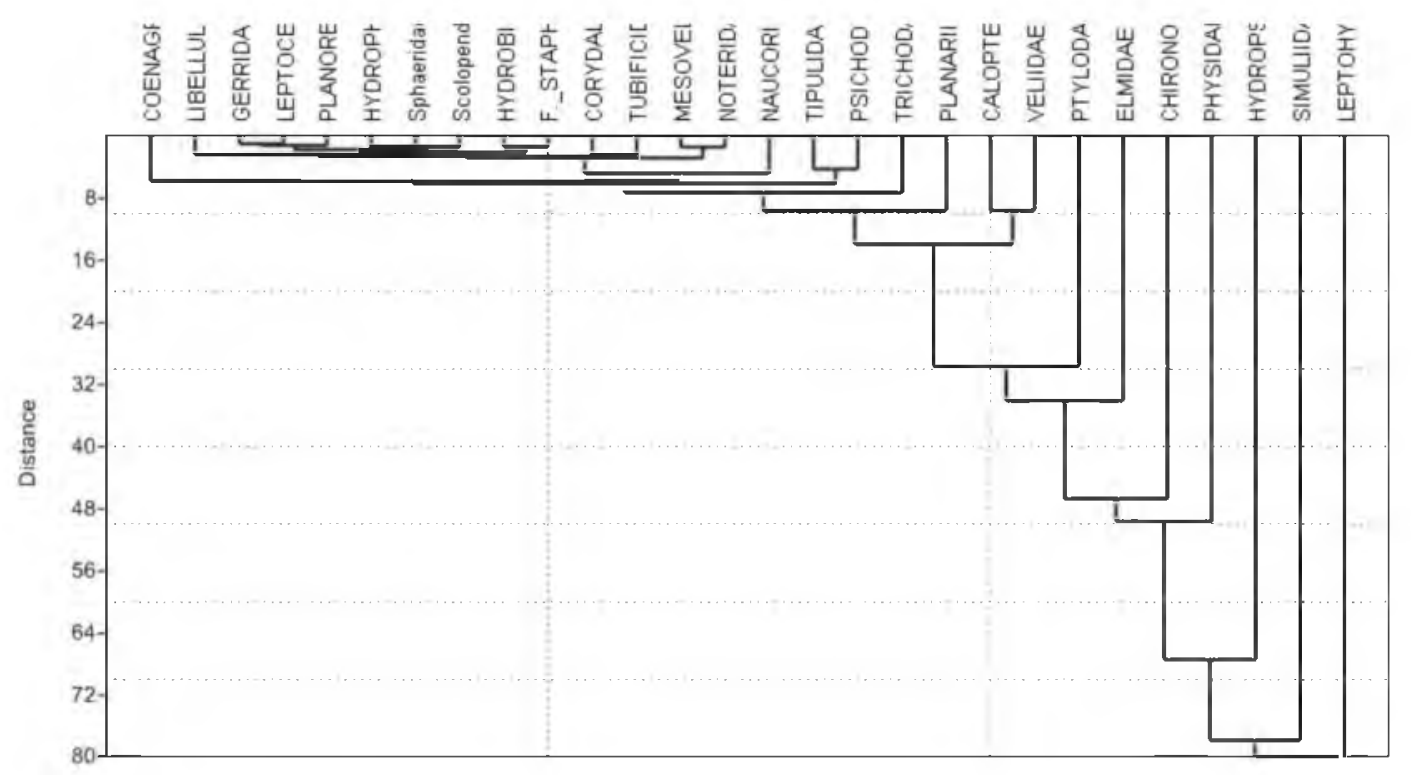

Figura 8. Dendrograma del análisis de Clúster (distancia euclidiana y ligamiento aritmético UPGMA). Distribución espacio-temporal de macroinvertebrados acuáticos en la quebrada La Cascajosa.

Mediante el análisis de conglomerados (Figura 8), se puede observar claramente el agrupamiento de tres conjuntos, el primer grupo caracterizado por aquellos taxa abundantes espacio-temporalmente, resaltando las familias Leptohyphidae, Hydropsychidae, Simuliidae, y Physidae. En segundo lugar por aquellos taxa que mostraron menores densidades y presencia limitada en todos los sitios de estudio, como Calopterygidae, Planariidae y Trichodactylidae y en menor grado Staphylinidae, Hydrobiosidae y Scolopendromorpha.

\section{Conclusiones}

La distribución de macroinvertebrados acuáticos en La quebrada La Cascajosa fue fluctuante debido posiblemente a las variaciones estacionales de los organismos, las cuales se presentan en función del efecto que ocasionan las lluvias o las sequías, que alteran el volumen de agua $y$ modifican la disponibilidad de hábitats.

Las Estaciones 1 y 2 de la quebrada La Cascajosa presentaron un comportamiento análogo posiblemente debido a su similitud en cuanto a fisiografía y paisaje, ya que las dos estaciones están ubicadas en zona rural.

Al analizar la variación de la abundancia de macroinvertebrados, se observó un ligero aumento en todos los sitios evaluados durante los meses en los que la lluvia fue escasa (noviembre 2011, mayo 2012). En las estaciones 2 y 3 de la quebrada La Cascajosa se determinó mayor abundancia relativa para las especies de macroinvertebrados que son catalogadas como tolerantes.
Se observó una diferencia significativa en la riqueza para la comunidad de macroinvertebrados, por estaciones de muestreo a lo largo de la quebrada La Cascajosa, posiblemente debido al gradiente altitudinal y a otros factores como el clima y para las estaciones 1, 2 y 3 presentó una Diversidad mediana de macroinvertebrados acuáticos según el índice de diversidad de Shannon y en la estación 4 la diversidad fue baja.

En la estación 2, se observó variación en la composición de macroinvertebrados acuáticos, debido a que el cauce de La quebrada recibe directamente los aportes de La quebrada la Cartagena y aguas abajo en la Estación 3, tiende a estabilizarse tanto la abundancia como la diversidad de especies, siguiendo el mismo patrón de la estación 1.

La estación 4 de la quebrada La Cascajosa, por estar altamente influenciada por actividades urbanas fue la más afectada, siendo la estación donde diferentes tipos de contaminantes tienen un efecto sinérgico sobre la comunidad bentónica, simplificando su estructura con una menor riqueza y diversidad de especies a lo largo de los meses muestreados.

De acuerdo con el BMWP, la estación 1 tiene aguas clase II, buena (aguas limpias), las demás estaciones presentan agua Clase III, aceptable (ligeramente contaminada).

En La quebrada La Cascajosa las características del lecho y la pendiente sean determinantes en la estructura de la comunidad de macroinvertebrados acuáticos ya que propician una moderada diversidad de hábitats para los macroinvertebrados, factores que incidieron en la mediana biodiversidad de especies registradas. 
Se pudo observar la tendencia de las dos estaciones urbanas (3 y 4) a presentar menores valores del $\mathrm{pH}$, indicando mayor acidez en la estación 4, debido a la mayor descarga de contaminantes en esta zona, lo que indica mantener el $\mathrm{pH}$ en condiciones ligeramente ácidas a través de los períodos muestreados. Los valores para la alcalinidad hallados indican, que La quebrada La Cascajosa es un ecosistema estable posiblemente influenciado por las bajas variaciones de $\mathrm{pH}$.

En La quebrada, el contenido de sólidos totales, tuvo una tendencia al aumento en su paso a través de la microcuenca. Así mismo, en la quebrada La Cascajosa se pudo observar que hay un aumento de nutrientes a través de las estaciones rurales a urbanas.

El análisis de componentes principales por correlación indicó que las estaciones 1, 2 y 3 se encuentran más correlacionadas en cuanto a la abundancia, diversidad y riqueza mostrando mayor porcentaje de similitud y la estación 1 y 2 con mayor correlación para la diversidad y riqueza de especies.

El análisis de Clúster indicó que los sitios que presentaron mayor similaridad fueron las estaciones 1 y 2 con un rango de similaridad por encima del $96 \%$. El análisis de conglomerados mostró un agrupamiento de tres conjuntos, el primer grupo caracterizado por aquellos taxa abundantes espacio-temporalmente, resaltando las familias Leptohyphidae, Hydropsychidae, Simuliidae, y Physidae. En segundo lugar por aquellos taxa que mostraron menores densidades y presencia limitada en todos los sitios de estudio, como los taxa Calopterygidae, Planariidae y Trichodactylidae y en menor grado Staphylinidae, Hydrobiosidae y Scolopendromorpha.

\section{Referencias bibliográficas}

BAUMGAERTNER, D.; MOERTL, M. y ROTHHAUPT, K. 2008. Effects of water - depth and water - level fluctuations on the macroinvertebrate community structure in the litoral zone of lake constance Hidrobiología 613:97-107.

BLANCO, J.P. 2002. Flujo de Nitrógeno y detritus en una quebrada de bosque seco tropical (Río Pesacador, Norte del Valle del Cauca). Actualidades Biológicas. 24 (76): 49- 58 .

BUSS, D. y BORGES, E. 2008. Aplication of rapid bioassessment protocols (RBP) for benthic macroinvertebrates in Brazil: Comparison betwen sampling techniques and mesh sizes. Neotrop. Entomol. 37:288 - 295.

CLAIR, T. y EHRMAN J. 1996. Variations in dischargue and DOC and N export from terrestrial basins with changues in climate: a neural network aproach. Limnol Oceanogr 41 (5): 921-927.

CHALARCA, D.; MEJÍA R.; AGUIRRE, J. 2007 Aproximación a la Determinación del Impacto de los Vertimientos de las Aguas Residuales Domésticas del Municipio de Ayapel,sobre la Calidad del Agua de la Ciénaga, Revista Facultad de Ingeniería Universidad de Antioquia, 40:41-58.

DOMINGUEZ, E. y FERNANDEZ, H. 2009. Macroinvertebrados Bentónicos sudamericanos. Sistémática y Biología. Edit. Fundación Miguel Lillo, Tucamán, Argentina. Tomo I. y II.

ESCUELA DE INGENIERIA DE RECURSOS NATURALES Y DEL AMBIENTE. AREA DE INGENIERIA SANITARIA Y AMBIENTAL. 2001 Caracterización puntual de los componentes físico-químicos, bacteriológicos e hidrobiológicos en el lago central de la Universidad del Valle. Facultad de Ingenieria. UNIVALLE, Cali. 41 p.

FLINT, O .1991. Studies of the neotropical caddisflies XLV. The taxonomy, phenology and faunistics of the Trichoptera of
Antioquia, Colombia. Smithsonian Contribution to Zoology. No. 520.

GUERRERO, F MANJARRES,A Y NUÑEZ, N. 2003. Los macroinvertebrados bentónicos de pozo azul, Cuenca del Río Gaira, Colombia y su relación con la calidad de agua. Acta Biol. Col. 8(2):43-55.

GRIMM, N., FAETH, S., GOLUBIEWSKI, N., REDMAN, C., WU, J., BAI, X., BRIGGS, J. 2008. Global change and the ecology of cities. Science 319, $756-760$.

HELLAWELL, J. M. 1989. Biological indicators of freshwater pollution and environmental management, Elsevier, Aplied Science Publ. London England. \& New York. 546p.

HOLDRIDGE, L.1996. Ecología basada en Zonas de Vida. Instituto Interamericano de Cooperación para la Agricultura. San José, Costa Rica. 78pp.

HUBBARD, M. 1990. Mayflies of the world. A Catalog of the family and genus group taxa. Flora and Fauna Handbook. 8: 1-115.

JACOBSEN, D.; CRESSA, C.; MATHOOKO, J. y DUDGEON D. 2008. Macroinvertebrates: Composition, Life Histories and Production. Tropical Stream Ecology. Pag, $65-105$.

KADLEC, R. y KNIGHT, R. 2001. Treatment Wetlands. CRC Press/Lewis Publishers, Boca Raton, FL... p. 893. En: Transformations of Nutrients in Natural and Constructed Wetlands. Editado por Jan Vymazal. Backhuys Publishers, Leiden, The Netherlands.

LAMPERT. W. AND SOMMER U. 1997. Limnoecology. The ecology of lakes and streams. Oxford University Press, NY USA. 382 p.

MACHADO, T. Y ROLDAN, G. 1981. Estudio de las características fisicoquímicas y biológicas del Río Anori y sus principales afluentes. Actualidades Biológicas, 10 (35) 3-19.

MARGALEF, R., 1991. Ecología. Omega, Barcelona. 951 págs.

MARTELLA, M. TRUMPER, E. BELLIS, L. RENISON, D. GIORDANO, P. BAZZANO, G. y GLEISER,R. 2012. Manual de Ecología y Evaluación de la biodiversidad. Reduca (Biología). Serie Ecología. 5 (1): 71-115, 2012. ISSN: 1989-3620.

MARTÍNEZ, M. 2009. Macroinvertebrados acuáticos como indicadores de calidad del agua en tres ciénagas del departamento de Cesar, Colombia. [Tesis de maestria] Bogotá: Departamento de Biología, Facultad de Ciencias, Universidad Nacional de Colombia.

MERRITT, W. y CUMMINS, K. 2008. An introduction to the aquatic insects of North American. Fourth edition. Kendall / Hunt Publishing Company. Printed in the United States of America. $1218 \mathrm{p}$.

METCALF, A. y EDDY, J. 1996. Ingeniería de aguas residuales: tratamiento, vertido y reutilización. Tomo I. McGraw-Hill/ Interamericana S.A. México D.F.

MINISTERIO DE SALUD. República de Colombia. 1984. Decreto 1594. Bogotá.

MONTOYA, Y. 2008. Colonización de sustratos rocosos por los macroinvertebrados acuáticos en La quebrada Los andes, El Carmen de Viboral, Antioquia Colombia. Revista Universidad Católica de Oriente. 23: 89 - 106.

PAVE, P. y MARCHESE, M. 2005 Invertebrados bentónicos como indicadores de calidad del agua en ríos urbanos (Paraná-Entre Ríos, Argentina). Ecol. austral [online]., vol.15, n.2 [citado 201206-28], pp. 183-197. Disponible en: <http://www.scielo.org.ar/scielo.php?script=sci_arttext\&pid=S1 667-782X2005000200008\&lng=es\&nrm=iso $>$. ISSN $1667-782 \mathrm{X}$

PÉREZ, A. y RODRIGUEZ, A. 2008. Índices fisicoquímicos de la calidad de agua para el manejo de lagunas tropicales de inundación. Rev. biol. trop. [serial online].

[citado 17 junio 2012] vol.56, no.4 p.1905-1918 [28 pantallas] Disponible en: URL:

$<$ http://www.scielo.sa.cr/scielo.php?script=sci_arttext\&pid $=$ SO0 34-77442008000400026\&lng=es\&nrm=iso

PLAN DE ORDENAMIENTO TERRITORIAL-URBANO (POT). DEL MUNICIPIO DE GARZÓN HUILA. 2007 Planeación Municipal. 2007.7v.

PONTIFICIA UNIVERSIDAD CATÓLICA DE ESMERALDAS. 2011. Evaluación Ambiental de los cantones Eloy Alfaro y San Lorenzo (Cuenca del Río Santiago y Bogotá) [en línea] [citado 19 -08-12] disponible en: www.senagua.gob.ec/files/informes/InformeInter MAESenagua.pdf

POSADA, J.; ROLDAN, G. y RAMÍREZ, J. 2000. Caracterización fisicoquímica y biológica de la calidad de aguas de la cuenca de la quebrada Piedras Blancas, Antioquia, Colombia. Rev BiolTrop.; 48(1):59-70.

RAMIREZ, G. y VIÑA, V. 1998. Limnología Colombiana. Editorial Panamericana, Forma e impreso S. A. Santafé de Bogotá. 293 p.

RIVERA, N. y MUÑOZ A. 1999 Parámetros químicos relevantes para el estudio de impacto ambiental en un río del sur de Chile. Información Tecnológica. 10 (5):91-101.

RIVERA, N., ENCINA F., MUÑ̃Z, A. Y MEJIA, P. 2004 La calidad de las 
aguas en los ríos Cautín e Imperial, IX Región-Chile. Información Tecnológica, $15(5), 89-101$.

RIVERA, J. 2011. Relación entre la composición y biomasa de la comunidad de macroinvertebrados acuáticos y las variables físicas y químicas en el humedal Jaboque Bogotá-Colombia. Maestría tesis, Universidad Nacional de Colombia. (Consultado 2012-04-11) Disponible en:

http://www.bdigital.unal.edu.co/4206/

RODIER, J. 1990 Análisis de las aguas. Ediciones OMEGA, S.A. Barcelona.

ROL.DAN, G. 1992. Fundamentos de Limnología Neotropical. Editorial Universidad de Antioquia. Medellín

1996. Guía para el estudio de los macroinvertebrados acuáticos del Departamento de Antioquia. Facultad de Ciencias exactas y naturales. ISBN 958-9129-02-8. Medellín Colombia.

1999. Los macroinvertebrados y su valor como indicadores de la calidad del agua. Revista de la Academia Colombiana de Ciencias exactas, físicas y naturales. Septiembre. 23(88).

2001. Los macroinvertebrados y su uso como indicadores de la calidad del agua. Memorias del taller "Medidas de la biodiversidad en Biología y Paleobiología", págs. 20-32

ROLDÁN, G.; BOHÓROUEZ, A.; CATAÑO, R.; ARDILA, J. 2001. Estudio limnológico del embalse del Guavio. Revista de la Academia Colombiana de ciencias exactas físicas y naturales. 24(90):25-33.

RUEDA, G. 2002. Manual de métodos en limnología. Editorial ACLLimnos, Bogotá, Colombia, $77 \mathrm{pp}$.

SEGNINI, S. 2003. El uso de los macroinvertebrados bentónicos como indicadores de la condición ecológica de los cuerpos de agua corriente. ECOTROPICOS 16(2):45-63.

SIERRA, C. 2011 . Calidad de agua. Evaluación y diagnóstico. $1^{\text {a }}$ edición. Universidad de Medellin. ISBN : 978-958-8692-06-07. Medellín Colombia. $456 \mathrm{p}$.

SILVA J. 2002. Humedales construidos. Compilador.[Serial online] [citado 7 Junio 2012]; [18 pantallas] Disponible en: URL:

http://www.bvsde.paho.org/bvsaar/e/fulltext/gestion/humedales.p df.

TEXEIRA, D. 1993. Caracterizäo limnológica dos sistemas lóticos e variacäo temporal e espacial de invertebrados bentónicos na bacia do ribeiräo do feijäo (Sáo Carlos - SP). Tesis de maestría. Escuela de ingeniería de Sáo Carlos. Brasil. 192 p.

TRISKA, F.; PRINGLE, C; ZELLWEGER, G.; DUFF, J. AND AVANZINO, R. 1993. Dissolved inorganic nitrogen composition, transformation and transport in naturally phosphate rich and phosphate-poor tropical streams. Can j. fish. Aquatic SCI 50:665-675.

WELCH, E.B. 1992. Ecologycal effects of waste water: Applied limnology and pollutant effects. Chapman \& Hall. Londres.

ZAMORA, H. 2007. El índice BMWP y la evaluación biológica de la calidad del agua en los ecosistemas acuáticos epicontinentales naturales de Colombia. Revista de la Asociación Colombiana de Ciencias Biológicas, 19.73-81. ISSN 0120-4173.

ZILLI, F. y GAGNETEN, A. 2005 .Efectos de la contaminación por metales pesados sobre la comunidad bentónica de la cuenca del arroyo cululú (río salado del norte, Argentina). INCI [online]., vol.30, n.3 ISSN 0378-1844, pp. 159-165 . [citado 2012-06-28] Disponible en:

http://www.scielo.org.ve/scielo.php?script=sci_arttext\&pid $=$ S0378-1 8442005000300009\&lng $=$ es \&nrm=iso. 